\title{
HD 74438: A tight spectroscopic quadruple as possible progenitor of sub-Chandrasekhar Type la supernovae
}

Thibault Merle ( $\boldsymbol{\nabla}$ tmerle@ulb.ac.be)

Université Libre de Bruxelles https://orcid.org/0000-0001-8253-1603

\section{Adrian Hamers}

Max-Planck-Institut für Astrophysik

Sophie Van Eck

Université Libre de Bruxelles

Alain Jorissen

Université Libre de Bruxelles

Mathieu Van der Swaelmen

INAF

Karen Pollard

University of Canterbury https://orcid.org/0000-0001-7442-4883

Dimitri Pourbaix

Université Libre de Bruxelles

Rodolfo Smiljanic

Nicolaus Copernicus Astronomical Center https://orcid.org/0000-0003-0942-7855

Tomaz Zwitter

University of Ljubljana

Gregor Traven

Lund Observatory

Gerard Gilmore

University of Cambridge https://orcid.org/0000-0003-4632-0213

Maria Sofia Randich

Osservatorio Astrofisico di Arcetri https://orcid.org/0000-0003-2438-0899

Anaïs Gonneau

University of Cambridge https://orcid.org/0000-0001-9091-5666

Anna Houriane

University of Cambridge

Germano Sacco

INAF

C. Worley 


\section{Letter}

Keywords: stars, sub-Chandrasekhar Type la supernova, stellar systems, spectroscopic quadruple

Posted Date: November 10th, 2021

DOI: https://doi.org/10.21203/rs.3.rs-1050702/v1

License: (c) (1) This work is licensed under a Creative Commons Attribution 4.0 International License. Read Full License

Version of Record: A version of this preprint was published at Nature Astronomy on May 12th, 2022. See the published version at https://doi.org/10.1038/s41550-022-01664-5. 


\section{HD 74438: A tight spectroscopic quadruple as possible progenitor of sub-Chandrasekhar Type la supernovae}

Thibault Merle ${ }^{1}$, ORCID 0000-0001-8253-1603

Institut d'Astronomie et d'Astrophysique, Université Libre de Bruxelles, CP. 226, Boulevard du Triomphe, 1050 Brussels, Belgium

Adrian S. Hamers

Max-Planck-Institut für Astrophysik, Karl-Schwarzschild-Strasse 1, 85741 Garching, Germany

Sophie Van Eck

Institut d'Astronomie et d'Astrophysique, Université Libre de Bruxelles, CP. 226, Boulevard du Triomphe, 1050 Brussels, Belgium

Alain Jorissen

Institut d'Astronomie et d'Astrophysique, Université Libre de Bruxelles, CP. 226, Boulevard du Triomphe, 1050 Brussels, Belgium

Mathieu Van der Swaelmen

INAF - Osservatorio Astrofisico di Arcetri, Largo E. Fermi, 5, 50125 Firenze, Italy

Karen Pollard

School of Physical and Chemical Sciences, University of Canterbury, Private Bag 4800, Christchurch 8140, New Zealand

Dimitri Pourbaix

FNRS, Institut d'Astronomie et d'Astrophysique, Université Libre de Bruxelles, CP. 226, Boulevard du Triomphe, 1050 Brussels, Belgium

Rodolfo Smiljanic

Nicolaus Copernicus Astronomical Center, Polish Academy of Sci-ences, ul. Bartycka 18, 00-716, Warsaw, Poland

Tomaž Zwitter

Faculty of Mathematics and Physics, University of Ljubljana, Jadranska 19, 1000, Ljubljana, Slovenia

Gregor Traven

Faculty of Mathematics and Physics, University of Ljubljana, Jadranska 19, 1000, Ljubljana, Slovenia

Gerry Gilmore

Institute of Astronomy, University of Cambridge, Madingley Road, Cambridge CB3 OHA, United Kingdom

Sofia Randich

INAF - Osservatorio Astrofisico di Arcetri, Largo E. Fermi 5, 50125, Florence, Italy

Anaïs Gonneau

Institute of Astronomy, University of Cambridge, Madingley Road, Cambridge CB3 OHA, United Kingdom

Anna Hourihane

Institute of Astronomy, University of Cambridge, Madingley Road, Cambridge CB3 OHA, United Kingdom

Germano Sacco

INAF - Osservatorio Astrofisico di Arcetri, Largo E. Fermi 5, 50125, Florence, Italy

C. Clare Worley

Institute of Astronomy, University of Cambridge, Madingley Road, Cambridge CB3 OHA, United Kingdom

${ }^{1}$ Corresponding author 
Stars often form in multiple systems and may follow a complex evolution involving mass transfer and collisions, leading to mergers that are possible progenitors of Type la supernovae ( $\mathrm{SNe}$ ) [1, 2]. The progenitors of such explosions are still highly debated [3]. While binaries have received much attention so far, higher-order stellar systems show a wide variety of interactions especially in tight systems, like long-term gravitational effects playing a key role in triple (where they are called von Zeipel-Lidov-Kozai , [4, 5], hereafter ZLK, oscillations) and quadruple systems. Here we report on the properties of the first spectroscopic quadruple (SB4) found within a star cluster: the $2+2$ hierarchical system HD 74438 [6]. Its membership in the open cluster IC 2391 makes it the youngest (43 My) SB4 discovered so far. The eccentricity of the $6 \mathrm{y}$ outer period is 0.46 and the two inner orbits, with periods of $20.5 \mathrm{~d}$ and $4.4 \mathrm{~d}$, and eccentricities of 0.36 and 0.15 , are not coplanar. Using an innovative combination of ground-based high resolution spectroscopy $[7,8,9,10]$ and Gaia/Hipparcos astrometry $[11,12,13,14]$, we show that this system is undergoing secular interaction that likely pumped the eccentricity of one of the inner orbit higher than expected for the spectral types of its components. We compute the future evolution of HD $\mathbf{7 4 4 3 8}$ by considering gravitational dynamics, stellar evolution, and binary interactions [15], and show that this system is an excellent candidate progenitor of sub-Chandrasekhar Type la supernova through white dwarf (WD) mergers. This specific type of SNla better accounts for the chemical evolution of iron-peak elements in the Galaxy [16].

The integrated spectral type of the HD 74438 system is A5 [17]; it belongs to one of the closest young clusters, IC 2391 containing 254 stars [18], located at $146^{+8}-7 \mathrm{pc}$ in the Vela constellation, aged $43^{+15}$ - My [19, 20] and of solar metallicity [21]. It was expected [22] that this system should at least be a triple star because it lies 0.9 mag above the main sequence (MS) of the Hertzsprung-Russell (HR) diagram of the parent cluster. It was detected as a four-component spectroscopic system (SB4) only recently [6] within the ground-based, large spectroscopic Gaia-ESO Survey (GES) [7, 8]. Follow-up observations with HRS/SALT [9] and HERCULES/UCMJO [10] allowed us to derive the orbital solution independently for the brighter (components $\mathrm{A}$ and $\mathrm{B}$ ) and the fainter (components $\mathrm{C}$ and D) pairs (Table 1 and Methods). The systemic velocities of each pair ( $v_{0}$ in Table 1$)$ bracket the velocity of the parent cluster [23] $\left(14.98 \pm 0.17 \mathrm{~km} \mathrm{~s}^{-1}\right)$, a first hint towards the pairs $A B$ and $C D$ being gravitationally bound. The component mass ratios imply similar brightnesses in each of the inner pairs, well in line with their detection as SB2s.

We adjusted synthetic spectra (see Methods) on two HRS/SALT spectra displaying four well-separated components. One of these observed spectra is shown in Fig. 1, together with the synthetic spectra of the four components and the resulting combined synthetic spectrum. The derived effective temperatures and spectral types are listed in Table 1.

Masses, luminosities and radii are then inferred from a stellar isochrone [24] of $43 \mathrm{My}$ at solar metallicity, since the system components necessarily lie (provided no mass transfer has occurred) on the cluster main sequence (see Fig. 2 and Methods). An important validation of our method is that the spectroscopic mass ratios thus derived are in good agreement, within the uncertainties, with the dynamical ones (Methods and Table 1). The dynamical mass ratio $q=$ $\left(M_{\mathrm{C}}+M_{\mathrm{D}}\right) /\left(M_{\mathrm{A}}+M_{\mathrm{B}}\right)=0.692 \pm 0.003$ could thus be derived, and appears to be among the lowest $q$ of SB4s known so far (see Supplementary Information). Adding together the four component 
luminosities, a total luminosity of $15.7 \pm 1.8 \mathrm{~L}_{\odot}$ is obtained, which is in excellent agreement with the independent determination provided by the Gaia DR2 luminosity [11] (15.3 ${ }_{-0.1}^{+0.2} \mathrm{~L}_{\odot}$, Fig. 2).

We can deduce, following Eqs. 1, 5 and 6 of Methods, the orbital inclinations with respect to the sky plane : $i_{\mathrm{AB}}=52.5 \pm 1.5^{\circ}$ for the massive pair and $i_{\mathrm{CD}}=84.0 \pm 0.9^{\circ}$ for the low-mass pair (Table 1). The two SB2 orbits are thus far from being coplanar. The CD pair should moreover show eclipses that could however not be detected from the inspection of the TESS [25] photometric data. Thanks to archival ESO observations and the recent RV follow-up, it became possible to constrain the mutual orbit of the two pairs. The period and eccentricity of the wide pair are found to be $5.7 \mathrm{y}$ and 0.46 (Methods and Table 1). Moreover, thanks to the knowledge of the masses, the inclination of the wide pair on the sky is found to be $73.2^{\circ} \pm 2.7^{\circ}$. However, the current mutual inclinations $\left(i_{\mathrm{AB} / \mathrm{AB}-\mathrm{CD}}\right.$ and $\left.i_{\mathrm{CD} / \mathrm{AB}-\mathrm{CD}}\right)$ are not known because they require the knowledge of the longitudes of the ascending nodes (Eq. (10) of Methods), which can only be determined by astrometric or interferometric measurements, should they be able to turn HD 74438 in a visual system. In the Methods section, we show that Hipparcos $[13,14]$ and Gaia $[11,12]$ astrometric data succeed in doing so for the outer orbit, giving access to the corresponding longitude of the ascending node. However, a similar application of the astrometric method to the inner orbits is impossible because of the closeness of the pairs, and in this case, even an interferometric imaging approach is challenging. This innovative combination of Hipparcos and Gaia astrometric data will be especially useful in the context of the forthcoming Gaia data releases.

Concerning its birth condition, HD 74438's properties may be compared to those of the much younger quadruple system discovered in the star-forming core Barnard 5 and consisting of a young protostar and three gravitationally-bound dense gas condensations [26]. The latter system has a much wider separation, but it is recognised that separations tend to decrease over time as the protostar grows in mass and dynamically interacts with the local gas reservoir [27]. These two systems thus represent different stages in the evolution of multiple-star systems within their birth environment.

HD 74438 is a rare case with a relatively short outer period of 5.7 y when compared with the other known 2+2 quadruples from the Multiple Star Catalogue [28]. Such systems are uncommon in OCs; actually, our system has the shortest outer period known so far in an OC, as shown in the left panel of Fig. 3, $\mu$ Ori [29] being the previous record holder (see also Supplementary Information). It is currently unclear whether this results from an observational bias or from the difficulty to form them [30]. Orbital shrinking caused by gas accretion and dynamical friction mechanisms that took place during core fragmentation [27] seems to be required to account for such short outer periods.

The high level of characterisation of this quadruple system allows us to use it as a test bench to get insight into the secular evolution of multiple hierarchical systems [31]. Long-term gravitational effects in which orbit-averaged torques change the orbital angular momenta and eccentricities play a key role in triple and quadruple systems; they can indeed drive the eccentricity to high values in quadruple systems. Despite its importance, the ZLK mechanism was ignored for many years, but was revived some 10-20 years ago for triple systems with the detection of the eccentric planet 16 Cyg B [32] or the close to perpendicular orbits in the Algol system $[33,34]$. The double astrometric binary $\mu$ Ori has also been reported to experience ZLK cycles [29]. Within triple systems, the inner and outer orbits exchange angular momentum, 
which leads to mutual inclination and eccentricity oscillations on timescales much longer than the orbital periods. The inner orbit eccentricities can reach very high values, thus leading to nearly radial motion: together with orbit shrinkage, it can lead to merging of a pair [31, 40, 41]. In quadruple systems, orbit-averaged torques also change the orbital angular momenta and eccentricity vectors, and ZLK effects are actually even more complex than in triples [42, 43, 44]. In 2+2 systems like HD 74438, mutual ZLK cycles can take place: each binary acts as a distant perturber on the other pair. Of particular interest is the fact that quadruple systems with mutual ZLK cycles have been proposed as one possible channel to SN la progenitors [1, 2, 45], since high eccentricities are easily reached.

In HD 74438, evidence for ZLK oscillations caused by the interaction between the CD pair and the AB-CD pair is given by the fact that the eccentricity of the CD pair ( 0.15 in a $4.4 \mathrm{~d}$ orbit) is higher than what is expected for SB2 (from the SB9 catalogue [46]) and doubly eclipsing binaries [47] of similar spectral types, as clearly shown on the bottom right panel of Fig. 3. Moreover the threshold period for the circularisation of binaries in clusters aged $43 \mathrm{My}$ is about 7-8 d [48, their Fig. 2], larger thus than the $4.4 \mathrm{~d}$ period of the CD pair. The CD pair should thus have been already circularized given the age of IC 2391 unless secular evolution prevented it, through the exchange of angular momentum between the outer AB-CD orbit and the CD one.

To explore the evolution of HD 74438 (up to $10 \mathrm{~Gy}$ in the future), we use the Multiple Stellar Evolution code [15] that takes into account a wide range of processes, most importantly gravitational dynamics, stellar evolution, and binary interactions such as mass transfer and common-envelope evolution. We employ a Monte-Carlo approach to sample a set of realisations of HD 74438 taking into account the observational uncertainties (see Methods). The first interesting result is the fact that ZLK oscillations do indeed occur in the CD pair (right panel of Fig. 4), due to angular momentum transfer between the CD and AB-CD pairs, for a very wide range of mutual orbital inclinations between these pairs. Incidentally, the merging of the $A B$ pair is even more probable (left panel of Fig. 4) because the ZLK timescale of AB is shorter than the one of CD (see Methods). Hence for systems like HD74438 one or more mergers occur in almost $50 \%$ of the simulated cases. We show in the Methods the relative fractions of all mergers in our simulations (collisions, as well as CE evolution). Collisions between main-sequence stars are common, as well as CE events involving giant stars. Also possible are WD-WD mergers. The latter could lead to Type la supernovae [49], although in the case of HD 74438 the combined mass, according to our simulations, does not exceed the Chandrasekhar mass of $1.44 \mathrm{M}_{\odot}$ (Fig. 5).

Former studies have shown that it is extremely difficult to obtain correct SNla rates from collisions of two WDs in dense stellar environments or resulting from a merger in binary systems [1, 49 and references therein]. There were expectations that the situation would be more favorable in triples thanks to head-on collisions triggered by secular evolution involving ZLK oscillations [50]. However, the rate of WD-WD collisions in triples has been shown to be too low to explain standard SNla rates [45, 51]. Higher-order systems like HD 74438 could solve the issue since the merger rate can be much higher in stellar quadruples compared to triples [2]. Even if our simulations result in sub-Chandrasekhar mass WD, it has been shown recently that between $70-85 \%$ of all SNla could be produced by mergers leading to the explosion of a sub-Chandrasekhar WD [16, 52]. The present discovery and characterisation of a benchmark 
spectroscopic quadruple involving low-mass stars contributes to shed light on an important channel potentially producing SNla explosions.

\section{Acknowledgments}

T.M. and S.v.E. are supported by a grant from the Fondation ULB. A.S.H. thanks the Max Planck Society for support through a Max Planck Research Group. T.M. acknowledges M.G. Perderson and T. Van Reeth for investigating the TESS data. T.M. acknowledges A. Tokovinin for explanations about the Multiple Star Catalogue. T.Z and G.T.. acknowledges financial support from the Slovenian Research Agency (research core funding No. P1-0188). G.T. acknowledges support by the Swedish strategic research programme eSSENCE, the project grant "The New Milky Way" from the Knut and Alice Wallenberg foundation and the grant 2016-03412 from the Swedish Research Council. Based on data products from observations made with ESO Telescopes at the La Silla Paranal Observatory under programme ID 188.B-3002. These data products have been processed by the Cambridge Astronomy Survey Unit (CASU) at the Institute of Astronomy, University of Cambridge, and by the FLAMES/UVES reduction team at INAF/Osservatorio Astrofisico di Arcetri. These data have been obtained from the Gaia-ESO Survey Data Archive, prepared and hosted by the Wide Field Astronomy Unit, Institute for Astronomy, University of Edinburgh, which is funded by the UK Science and Technology Facilities Council. This work was partly supported by the European Union FP7 programme through ERC grant number 320360 and by the Leverhulme Trust through grant RPG-2012-541. We acknowledge the support from INAF and Ministero dell' Istruzione, dell' Universitl'a' e della Ricerca (MIUR) in the form of the grant "Premiale VLT 2012". The results presented here benefit from discussions held during the Gaia-ESO workshops and conferences supported by the ESF (European Science Foundation) through the GREAT Research Network Programme. Some of the observations reported in this paper were obtained with the Southern African Large Telescope (SALT) under program 2018-1-MLT-009 (PI: R. Smiljanic). Polish participation in SALT is funded by grant No. MNiSW DIR/WK/2016/07. This work has made use of data from the European Space Agency (ESA) mission Gaia (https://www.cosmos.esa.int/gaia), processed by the Gaia Data Processing and Analysis Consortium (DPAC, https://www.cosmos.esa.int/web/gaia/dpac/consortium). Funding for the DPAC has been provided by national institutions, in particular the institutions participating in the Gaia Multilateral Agreement. This work has made use of the VALD database, operated at Uppsala University, the Institute of Astronomy RAS in Moscow, and the University of Vienna. This research has made use of the SIMBAD database, operated at CDS, Strasbourg, France. This research has made use of the VizieR catalogue access tool, CDS, Strasbourg, France (DOI : 10.26093/cds/vizier). The original description of the VizieR service was published in A\&AS 143, 23. This research has made use of Python3 and IPython, and modules NumPy, SciPy and Pandas. All the graphics were generated with Matplotlib.

\section{References}

[1] Hamers, A. S., On the rates of Type la supernovae originating from white dwarf collisions in quadruple star systems, Mon. Not. R. Astron. Soc., 478, 620 (2018) 2018MNRAS.478.620H

[2] Fang, X., Thompson, T. A. \& Hirata, C. M., Dynamics of quadruple systems composed of two binaries: stars, white dwarfs, and implications for la supernovae, Mon. Not. R. Astron. Soc, 476, 4234 (2018) 2018MNRAS.476.4234F

[3] Maoz, D. et al., Observational Clues to the Progenitors of Type la Supernovae, Ann. Rev. Astron. Astrophys., 2014ARA\&A.52.107M

[4] Lidov, M.L. The evolution of orbits of artificial satellites of planets under the action of gravitational perturbations of external bodies, Planetary and Space Science, 9, 719 (1962), 1962P\&SS....9..719L

[5] Kozai, Y., Secular perturbations of asteroids with high inclination and eccentricity, Astron. J., 67, 591 (1962) $1962 \mathrm{AJ} \ldots . . .67 .591 \mathrm{~K}$

[6] Merle, T. et al., The Gaia-ESO Survey: double-, triple-, and quadruple-line spectroscopic binary candidates, Astron. Astrophys., 608, 95 (2017) 2017A\&A...608A..95M

[7] Gilmore, G. et al., The Gaia-ESO Public Spectroscopic Survey, The Messenger, 147, 25 (2012) 2012Msngr. 147...25G

[8] Randich, S. et al., The Gaia-ESO Large Public Spectroscopic Survey, The Messenger, 154, 47, (2013) 2013Msngr.154...47R

[9] Crause, L. A. et al., Performance of the Southern African Large Telescope (SALT) High Resolution Spectrograph (HRS), Proc. SPIE, 9147, 6TC (2014) 2014SPIE.9147E..6TC

[10] Hearnshaw, J. B. et al., The HERCULES Echelle Spectrograph at Mt John, Exp. Astron., 13, 59 (2002) 2002ExA....13 ...59H

[11] Gaia collaboration et al., Gaia Data Release 2. Summary of the contents and survey properties, Astron. Astrophys., 616, A1 (2018) 2018A\&A.616A..1G

[12] Gaia collaboration, Gaia Early Data Release 3. Summary of the contents and survey properties, Astron. Astrophys., 649, 1 (2021) 2021A\&A...649A...1G

[13] ESA, The HIPPARCOS and TYCHO catalogues. Astrometric and photometric star catalogues derived from the ESA HIPPARCOS Space Astrometry Mission, ESA Publications Division, 1200 (1997) 1997ESASP1200....E 
[14] Høg, E., et al., The Tycho-2 catalogue of the 2.5 million brightest stars, Astron. Astrophys., 355, 27 (2000) 2000A\&A...355L..27H

[15] Hamers, A.S., et al., Multiple Stellar Evolution: a population synthesis algorithm to model the stellar, binary, and dynamical evolution of multiple-star systems, Mon. Not. R. Astron. Soc., 502, 4479 (2021) 2021MNRAS.502.4479H

[16] Eitner, P. et al., Observational constraints on the origin of the elements III. Evidence for the dominant role of sub-Chandrasekhar SN la in the chemical evolution of Mn and Fe in the Galaxy, Astron. Astrophys., 635, A38 (2020) 2020A\&A...635A..38E

[17] Houk, N., Michigan catalogue of two-dimensional spectral types for the HD stars, Ann Arbor : Dept. of Astronomy (1978) 1978mcts.book.....H

[18] Gaia collaboration et al., Gaia Data Release 2. Observational Hertzsprung-Russell diagrams, Astron. Astrophys., 616, 10 (2018) 2018A\&A...616A..10G

[19] Randich, S. et al. The Gaia-ESO Survey: open clusters in Gaia-DR1. A way forward to stellar age calibration, Astron. Astrophys., 612, A99 (2018) 2018A\&A...612A..99R

[20] Gaia collaboration et al., Gaia Data Release 2. Summary of the contents and survey properties, Astron. Astrophys., 616, A1 (2018) 2018A\&A...616A...1G

[21] Spina, L. et al., The Gaia-ESO Survey: the present-day radial metallicity distribution of the Galactic disc probed by pre-main-sequence clusters, Astron. Astrophys., 601, 70 (2017) 2017A\&A...601A..70S

[22] Platais, I. et al., WIYN open cluster study. XXVI. Improved kinematic membership and spectroscopy of IC 2391, Astron. Astrophys. , 461, 509 (2007) 2007A\&A...461..509P

[23] Bravi, L. et al., The Gaia-ESO Survey: a kinematical and dynamical study of four young open clusters, Astron. Astrophys., 615, 37 (2018), 2018A\&A...615A..37B

[24] Bressan, A. et al., PARSEC: stellar tracks and isochrones with the PAdova and TRieste Stellar Evolution Code, Mon. Not. R. Astron. Soc., 427, 127 (2012) 2012MNRAS.427..127B

[25] Ricker, G. R. The Transiting Exoplanet Survey Satellite (TESS): Discovering New Earths and Super-Earths in the Solar Neighborhood, Americ. Astron. Soc., ESS meeting \#3, 47, 503, (2015) 2015ESS....350301R

[26] Pineda, J. E. et al. The formation of a quadruple star system with wide separation, Nature, 518, 213, (2015) 2015Natur.518.213P

[27] Lee, A. T. et al., The Formation and Evolution of Wide-orbit Stellar Multiples In Magnetized Clouds, Astrophys. J., 887, 232 (2019) 2019ApJ...887..232L

[28] Tokovinin, A., The Updated Multiple Star Catalog, Astrophys. J. Supp. S., 235, 6 (2018) 2018ApJS..235...6T

[29] Muterspaugh, M. W. et al., Masses, Luminosities, and Orbital Coplanarities of the $\mu$ Orionis Quadruple-Star System from Phases Differential Astrometry, Astron. J., 135, 766 (2008) 2008AJ...135..766M

[30] Tokovinin, A., From Binaries to Multiples. II. Hierarchical Multiplicity of F and G Dwarfs, Astron. J., 147, 87 (2014) 2014AJ....147...87T

[31] Naoz, S. The Eccentric Kozai-Lidov Effect and Its Applications, Ann. Rev. Astron. Astrophys., 54, 441 (2016) 2016ARA\&A..54...441N

[32] Cochran, W. D. et al., Detection of a planetary companion to 16 Cygni B, Bull. Am. Astron. Soc., 28, 1111 (1996) 1996BAAS...28.1111C

[33] Eggleton, P. P. et al., The Equilibrium Tide Model for Tidal Friction, Astrophys. J., 499, 853 (1998) 1998ApJ...499..853E

[34] Baron, F. et al., Imaging the Algol Triple System in the H Band with the CHARA Interferometer, Astrophys. J., 752, 20 (2012) 2012ApJ...752...20B

[40] Naoz, S. et al., Secular dynamics in hierarchical three-body systems, Mon. Not. R. Astron. Soc, 431, 2155 (2013) 2013MNRAS.431.2155N

[41] Li, G. et al., Eccentricity Growth and Orbit Flip in Near-coplanar Hierarchical Three-body Systems, Astron. J., 785, 116 (2014) 2014ApJ...785..116L

[42] Pejcha, O. et al., Greatly enhanced eccentricity oscillations in quadruple systems composed of two binaries: implications for stars, planets and transients, Mon. Not. R. Astron. Soc., 431, 943 (2013) 2013MNRAS.435..943P

[43] Hamers, A. S. et al., Secular dynamics of hierarchical quadruple systems: the case of a triple system orbited by a fourth body, Mon. Not. R. Astron. Soc., 449, 4221 (2015) 2015MNRAS.449.4221H

[44] Hamers, A.S. \& Lai, D., Secular chaotic dynamics in hierarchical quadruple systems, with applications to hot Jupiters in stellar binaries and triples, Mon. Not. R. Astron. Soc., 470, 1657 (2017) 2017MNRAS.470.1657H

[45] Hamers, A. S. et al., Population synthesis of triple systems in the context of mergers of carbon-oxygen white dwarfs, Mon. Not. R. Astron. Soc., 430, 2262 (2013) 2013MNRAS.430.2262H

[46] Pourbaix, D. et al., $\mathrm{S}_{\mathrm{B}}{ }^{9}$ : The ninth catalogue of spectroscopic binary orbits, Astron. Astroph., 424, 727 (2004)

2004A\&A...424..727P 
[47] Zasche, P. et al., Doubly eclipsing systems, Astron. Astrophys., 630, 128 (2019) 2019A\&A...630A.128Z

[48] Geller, A. M. et al., Direct N-body Modeling of the Old Open Cluster NGC 188: A Detailed Comparison of Theoretical and Observed Binary Star and Blue Straggler Populations, Astron. J., 145, 8 (2013) 2013AJ...145...86

[49] Wang, B. \& Han, Z., Progenitors of type la supernovae, New Astron. Rev., 56, 122 (2012) 2012NewAR..56..122W

[50] Katz, B. \& Dong, S., The rate of WD-WD head-on collisions may be as high as the SNe la rate, ArXiv (2012) 2012arXiv1211.4584K

[51] Toonen, S. et al., Rate of WD-WD head-on collisions in isolated triples is too low to explain standard type la supernovae, Astron. Astrophys., 610, 22 (2018) 2018A\&A...610A..22T

[52] Flörs, A. et al., Sub-Chandrasekhar progenitors favoured for Type la supernovae: evidence from late-time spectroscopy, Mon. Not. R. Astron. Soc, 491, 2902 (2020) 2020MNRAS.491.2902F

\section{Figures}

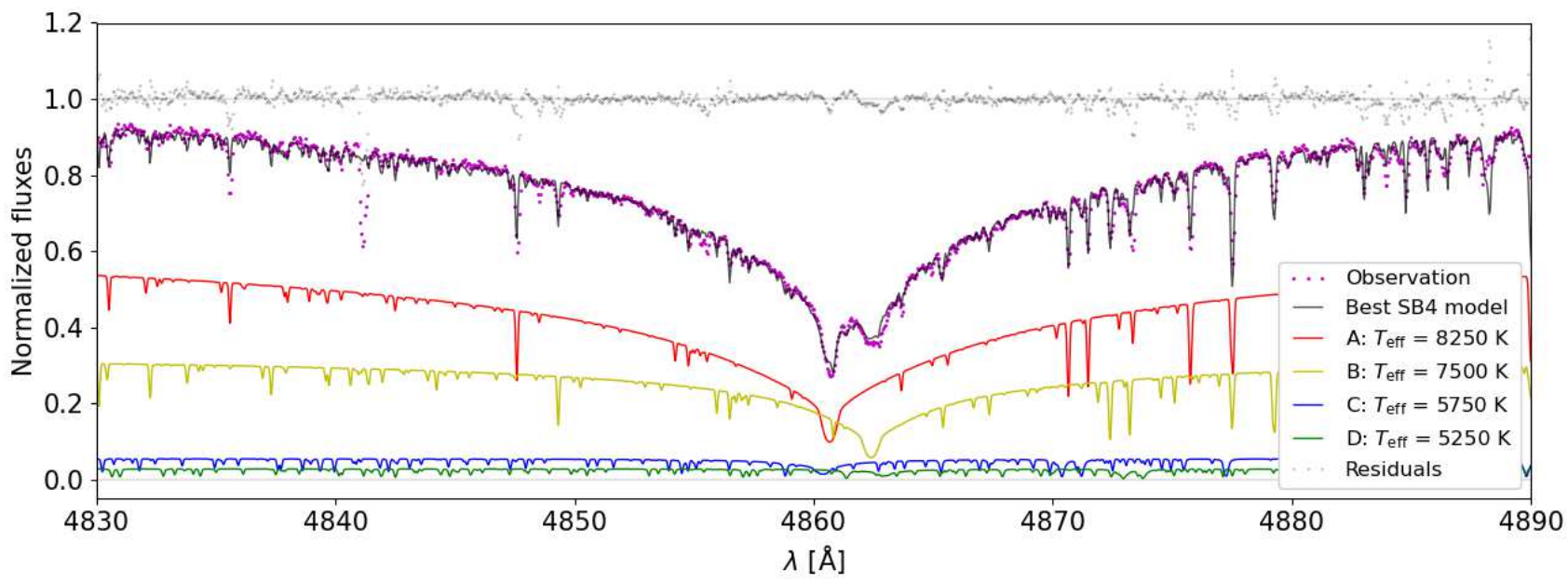

Figure 1: Spectral fitting of the HRS/SALT spectra (taken on 2018-12-31). The observed spectrum is shown as magenta dots and the best SB4 template is shown as a black solid line. The four individual component-synthetic spectra are displayed as labelled in the legend. For components $C$ and $D$, the temperatures adopted in this figure correspond to those of the closest models in the grid of step $250 \mathrm{~K}$. The residuals (observed-synthetic) are shown at the top. The strong line close to the middle is the Balmer line $H \beta$. 


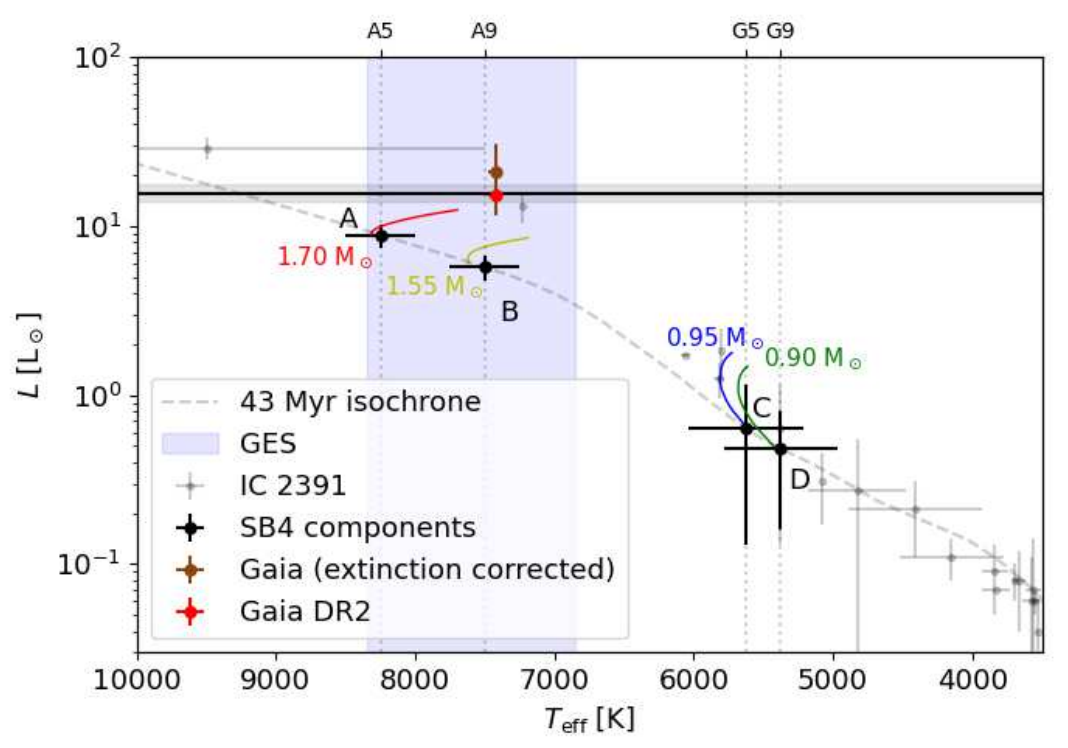

Figure 2: Location of HD 74438 in the HR diagram. Black dots mark the positions of the four individual components placed on the main sequence (dashed grey line), with $T_{\text {eff }}$ as derived from spectral fitting (vertical dotted lines with associated spectral type on top), to be compared with the temperature of the unresolved system derived by the Gaia-ESO Survey (the blue shaded area representing the $1 \sigma$ confidence interval). The combined luminosity derived in this work is plotted as the horizontal black line while the Gaia luminosities and temperatures of the unresolved system are indicated by red (raw) and brown (extinction-corrected) dots. The dashed grey line and colored lines are the theoretical stellar isochrone at $43 \mathrm{My}$ and for illustrative purposes the evolutionary tracks [24] for masses close to the derived ones. Grey dots are known members of the IC 2391 open cluster [19].
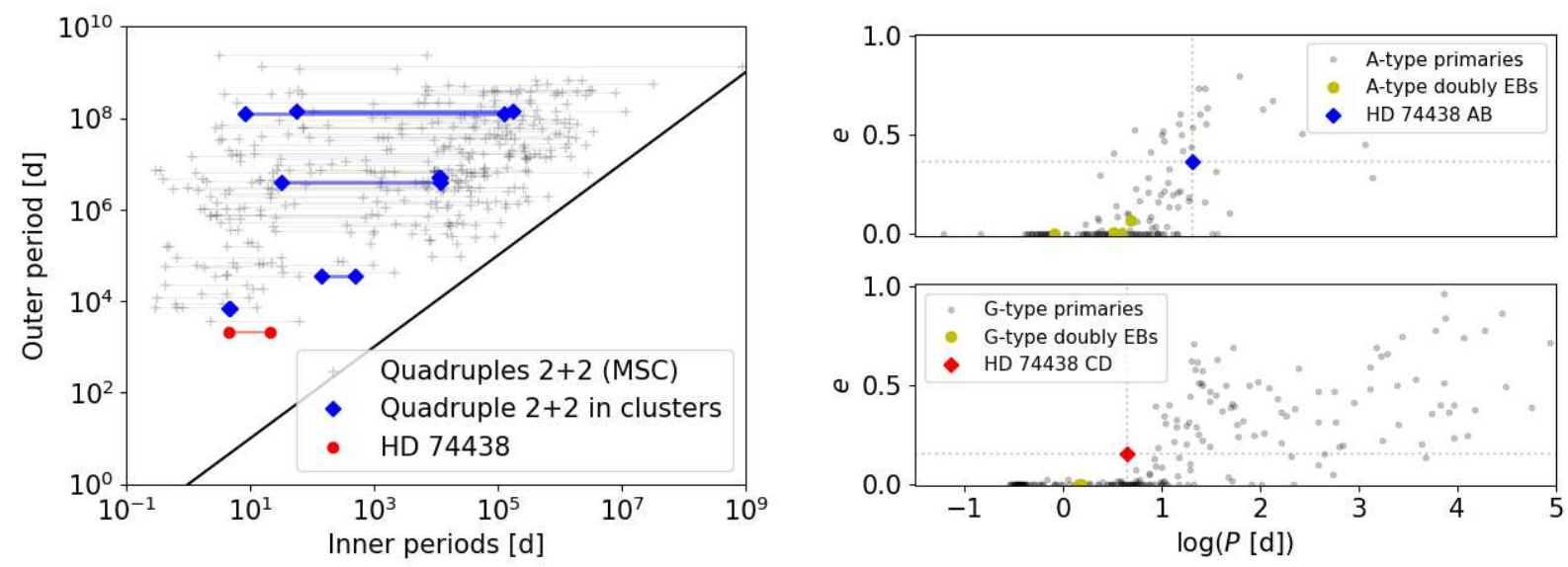

Figure 3. Left: Comparison with other known $2+2$ quadruples in clusters. Grey plusses are the $2+2$ quadruples from the Multiple Star Catalogue [58, not volume complete]. We highlighted blue quadruples belonging to clusters as cross-matched with SIMBAD. HD74438 appears to have the smallest outer period. The solid line represents the condition for $2+2$ quadruples to be dynamically stable [5]. Right: Location of the two inner SB2 (AB in blue, CD in red) in the eccentricity-period diagram, compared with A-type primaries (matching the AB pair; grey dots in the top panel) and G-type primaries (matching the CD pair; grey dots in the bottom panel) of the SB9 catalogue [59] of spectroscopic binaries and doubly eclipsing binaries [60] in yellow circles. 

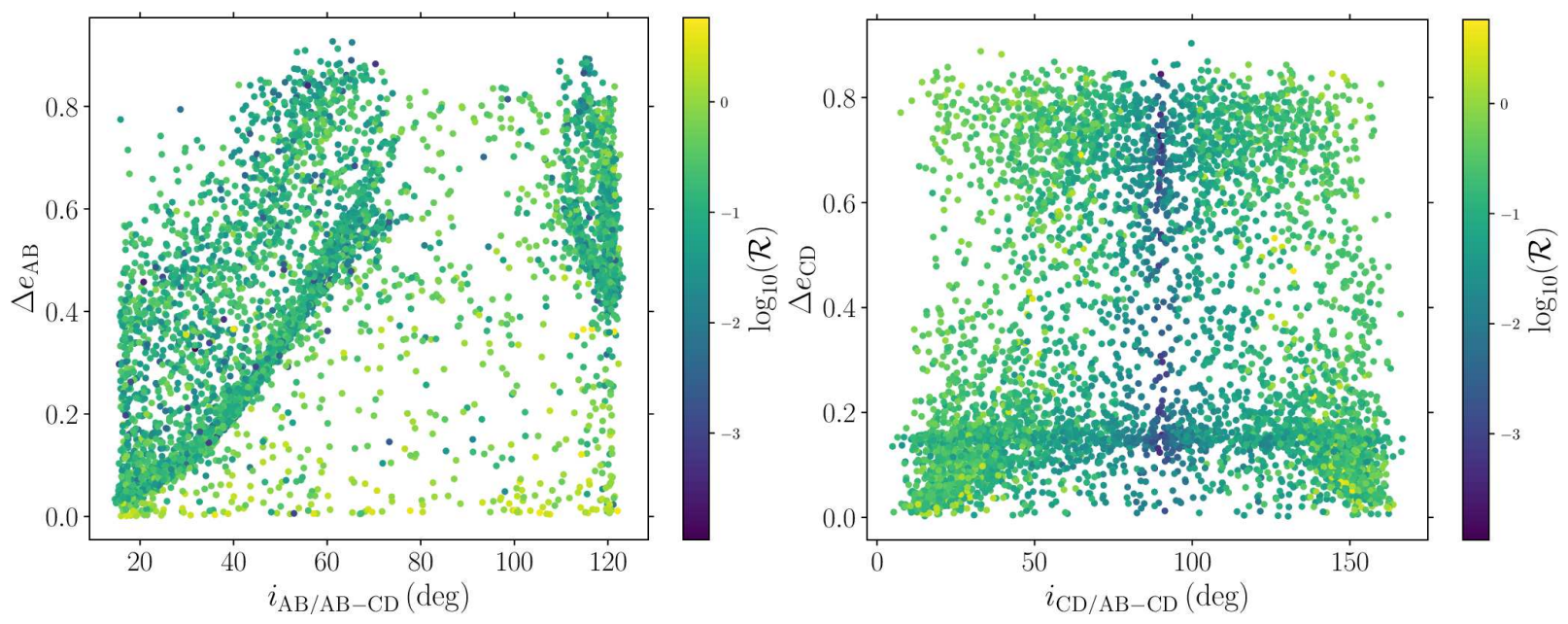

Figure 4. MSE simulations of the HD 74438 future. The figures show, for the surviving systems, the amplitude of eccentricity oscillations during the integrated time (i.e., up to $10 \mathrm{~Gy}$ ) as a function of the initial mutual inclination. Colour-coded are the initial values of $R$ (the ZLK time-scales for inner-to-outer orbit pairs; Eq. 32 of [45]). In the case of the $e_{A B}$ amplitude, there is a large gap near $90^{\circ}$ inclinations, since these have merged during their previous evolution. The $e_{C D}$-plot shows that high eccentricity amplitudes

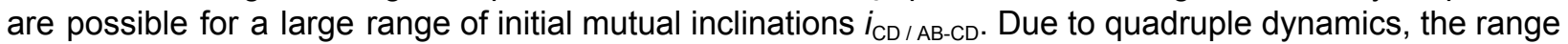
of mutual inclination angles is much enhanced, as can be seen by noting the colours: $R$ 's close to unity are required to get high amplitudes for small mutual inclinations, whereas if $i_{\mathrm{CD} / \mathrm{AB}-\mathrm{CD}}$ is close to $90^{\circ}$, high amplitudes can also be attained regardless of $R$ values.

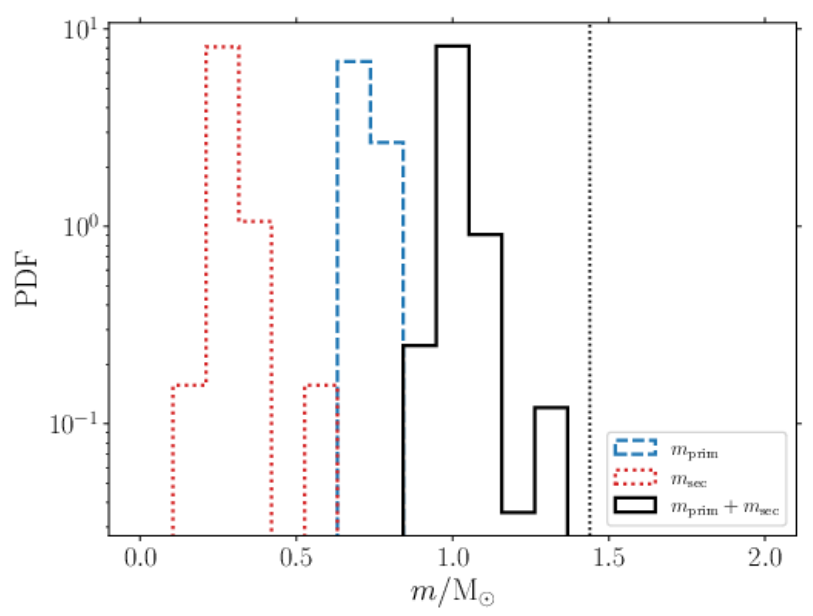

Figure 5. Distribution of merging WDs in our simulations. The total mass of the two merging WDs (solid black line), as well as the individual two WD masses resulting, most of the time, from the merger of the $A B$ pair (blue dashed) and $C D$ pair (red dotted lines). The black vertical dotted line indicates the Chandrasekhar mass of $1.44 \mathrm{M}_{\text {. }}$. 


\begin{tabular}{|c|c|c|c|c|c|c|}
\hline Component & A & B & C & D & \multicolumn{2}{|c|}{ Unresolved } \\
\hline Spectral Type & A5 & A9 & G5 & G9 & & \\
\hline$T_{\text {eff }}[\mathrm{K}]$ & $8250 \pm 250$ & $7500 \pm 250$ & $5625 \pm 410$ & $5375 \pm 410$ & & \\
\hline$M\left[M_{\odot}\right](a)$ & $1.70 \pm 0.06$ & $1.54 \pm 0.06$ & $0.96 \pm 0.14$ & $0.87 \pm 0.14$ & 5.07 & 0.22 \\
\hline$L\left[\mathrm{~L}_{\odot}\right]$ & $8.87 \pm 1.40$ & $5.72 \pm 0.95$ & $0.64 \pm 0.51$ & $0.48 \pm 0.32$ & 15.71 & $=1.80$ \\
\hline \multirow[t]{2}{*}{$R\left[R_{\odot}\right]$} & $1.46 \pm 0.15$ & $1.42 \pm 0.15$ & $0.84 \pm 0.36$ & $0.80 \pm 0.29$ & & \\
\hline & \multicolumn{2}{|c|}{$A B$} & \multicolumn{2}{|c|}{ CD } & \multicolumn{2}{|c|}{$A B-C D$} \\
\hline$q_{\text {spec }}$ & \multicolumn{2}{|c|}{$0.91 \pm 0.05$} & \multicolumn{2}{|c|}{$0.91 \pm 0.22$} & \multicolumn{2}{|c|}{$0.58 \pm 0.11$} \\
\hline$P[\mathrm{~d}]$ & \multicolumn{2}{|c|}{$20.56729 \pm 0.00008$} & \multicolumn{2}{|c|}{$4.424198 \pm 0.000006$} & \multicolumn{2}{|c|}{$2074.2 \pm 3.5$} \\
\hline e & \multicolumn{2}{|c|}{$0.3647 \pm 0.0002$} & \multicolumn{2}{|c|}{$0.1536 \pm 0.0004$} & \multicolumn{2}{|c|}{$0.458 \pm 0.015$} \\
\hline$\omega[\mathrm{rad}](\mathrm{c})$ & \multicolumn{2}{|c|}{$1.8901 \pm 0.0005$} & \multicolumn{2}{|c|}{$4.3612 \pm 0.0014$} & \multicolumn{2}{|c|}{$0.185 \pm 0.039$} \\
\hline$T_{0}-2400000[\mathrm{~d}]$ & \multicolumn{2}{|c|}{$58215.17 \pm 0.002$} & \multicolumn{2}{|c|}{$58290.76 \pm 0.001$} & \multicolumn{2}{|c|}{$59165.8 \pm 5.1$} \\
\hline$v_{0}\left[\mathrm{~km} \mathrm{~s}^{-1}\right]$ & \multicolumn{2}{|c|}{$8.371 \pm 0.007$} & \multicolumn{2}{|c|}{$23.724 \pm 0.017$} & \multicolumn{2}{|c|}{$14.5 \pm 0.2$} \\
\hline & & & & & $A B$ & CD \\
\hline$K\left[\mathrm{~km} \mathrm{~s}^{-1}\right]$ & $45.80 \pm 0.09$ & $50.88 \pm 0.09$ & $83.1 \pm 0.1$ & $85.3 \pm 0.1$ & $12.8 \pm 0.3$ & $18.5 \pm 0.4$ \\
\hline$q_{\mathrm{dyn}}$ & \multicolumn{2}{|c|}{$0.900 \pm 0.002$} & \multicolumn{2}{|c|}{$0.974 \pm 0.002$} & \multicolumn{2}{|c|}{$0.692 \pm 0.003$} \\
\hline$i\left[^{\circ}\right]$ & \multicolumn{2}{|c|}{$52.5 \pm 1.5$} & \multicolumn{2}{|c|}{$84.0 \pm 0.9$} & \multicolumn{2}{|c|}{$73.2 \pm 2.7$} \\
\hline$a[a u]$ & \multicolumn{2}{|c|}{$0.21 \pm 0.25$} & \multicolumn{2}{|c|}{$0.068 \pm 0.004$} & \multicolumn{2}{|c|}{$5.54 \pm 0.04$} \\
\hline$M\left[\mathrm{M}_{\odot}\right](\mathrm{b})$ & $1.64 \pm 0.06$ & $1.48 \pm 0.06$ & $1.09 \pm 0.04$ & $1.06 \pm 0.04$ & \multicolumn{2}{|c|}{$5.28 \pm 0.10$} \\
\hline $\begin{array}{l}\mu^{\prime \prime}{ }_{A B-C D, p h o t} \\
\text { [mas/y] }\end{array}$ & - & - & - & - & \multicolumn{2}{|c|}{13.0} \\
\hline$\Omega\left[{ }^{\circ}\right]$ & \multicolumn{2}{|c|}{$?$} & ? & & & $3^{\circ}$ \\
\hline
\end{tabular}

(a) Masses derived from the stars' location in the HR diagram

(b) Masses derived from Eqs. (5) and (6), adopting the value of $M_{A}$ from (a).

(c) The argument of periastron $\omega$ corresponds to the spectroscopic orbit of the brightest component around the centre of mass.

Table 1. Stellar and orbital parameters for the quadruple system HD 74438 and its components. 


\section{Methods}

Observations and data reduction. The details of the observations are summarized in Table 2. The spectra were obtained with three different high-resolution spectrographs: (i) UVES/VLT, (ii) HERCULES/UCMJO, (iii) HRS/SALT, and with one medium-resolution spectrograph (GIRAFFE/VLT).

UVES spectra have been obtained in the context of the Gaia-ESO Survey. HD 74438 has been observed 45 times within $2.5 \mathrm{~h}$ on the night of February 18-19, 2014, with the UVES/FLAMES multi-fiber facility [53] using the U520 and U580 set-ups. The four components of HD 74438 were detected in the framework of a search for spectroscopic binaries [6] within the GES sample. The spectra were reduced and normalized using the GES standard reduction pipeline [54].

A long-term monitoring program of HD 74438 has been undertaken at the University of Canterbury Mt John Observatory in New Zealand with the HERCULES spectrograph [10]. The spectra were reduced and normalized with a MATLAB software pipeline developed specifically for HERCULES spectra.

Finally, a monitoring program (no. 2018-1-MLT-009 and 2020-2-MLT-003) was accepted on SALT [9] (Southern African Large Telescope) to follow with the HRS spectrograph the most interesting SB2, SB3 and the SB4 candidates uncovered within the GES [6]. The spectra were reduced $^{2}$ with the SALT Science pipeline [55] and the continuum normalization was obtained by iteratively fitting polynomials and rejecting the residuals exceeding 0.25 standard deviations.

In addition, archival data from ESO with the mid-resolution spectrograph GIRAFFE/VLT [53] allows us to add three epochs in 2004 and help to constrain the longer period but only for the $A B$ pair because $C$ and $D$ components are not resolved with GIRAFFE.

Cross correlation function (CCF) calculations. The CCFs were computed by cross-correlating normalized observed spectra with a unique template built as follows. The initial guess for the template was based on the spectral type attributed to the unresolved multiple system (A2 [17]). We thus adopted a Kurucz model atmosphere [56] with $T_{\text {eff }}=9000 \mathrm{~K}$. Nevertheless a lower temperature $\left(T_{\text {eff }}=7000 \mathrm{~K}\right)$ provided more contrasted CCFs. Therefore we adopted a Kurucz model atmosphere of $T_{\text {eff }}=7000 \mathrm{~K}, \log g=4.0$ and $[\mathrm{Fe} / \mathrm{H}]=0$. We computed a synthetic spectrum from 3700 to $8900 \AA$ to cover the three spectrographs spectral range. Strong lines were masked (Balmer lines, H\&K Ca II, Ca II IR triplet, and Na I D lines). We then used Detection of Extrema (DOE) [6] to extract the positions and depths of 6123 lines, building a comb of rectangular functions $0.01 \AA$-wide, and with a height corresponding to the line depth. The CCFs were then computed by cross-correlating the normalized spectra and the comb of rectangular functions.

\footnotetext{
${ }^{2}$ http://pysalt.salt.ac.za/
} 
Radial-velocity measurements. The identification of the number of radial velocity (RV) components, their positions and the RV uncertainties was performed with DOE [6]. First, the third derivative of the CCF was used to identify the number of components. Second, a multi-Gaussian fit was performed on the CCF. Examples of fits to multiple CCF peaks are illustrated in Fig. 6 for HRS/SALT and HERCULES/UCMJO. Three components are always visible and a fourth one can also be distinguished in most CCFs; they are labelled A, B, C, D by decreasing CCF peak intensity. While the assignment of the $A$ and $B$ components is straightforward, several trials and errors were necessary for a correct assignment of the $C$ and D components because their peak intensities are similar, especially among the HRS spectra where the sparse sampling does not allow to easily follow each component over time.

Orbital parameters of the inner pairs. The HD 74438 system is a $2+2$ hierarchical system. The time interval between UVES and HERCULES/UCMJO + HRS/SALT data is about $5 \mathrm{y}$, enough for the wider $A B-C D$ pair to imprint a secular trend; therefore only the HERCULES/UCMJO and HRS/SALT data sets were used to derive the AB and CD short-period orbits. We also assumed that the instrumental zero-point RV offset between the two spectrographs is negligible. In Fig. 7, the final orbital solutions are compared to the HRS/SALT (middle bottom) and HERCULES/UCMJO (bottom) RVs. The symmetric $\pm 10 \mathrm{~km} \mathrm{~s}^{-1}$ offsets in the GES data are well visible in Fig. 8, which displays the orbital solutions for pairs AB (left panel, $P \approx 20.6 \mathrm{~d}$ ) and $\mathrm{CD}$ (right panel, $P \approx 4.4 \mathrm{~d}$ ), obtained from the HRS/SALT and HERCULES/UCMJO RVs. Orbital parameters of the two short-period pairs $A B$ and $C D$ are provided in Table 1.

Astrophysical parameters. The astrophysical parameters were derived using two HRS/SALT spectra taken on October 14, 2018 and December 31, 2018 because they show four well-separated components at the highest resolution. Since HD 74438 belongs to a young cluster, it is assumed to be located on the main sequence and to have a solar metallicity [21] (i.e. $\log g=4.5$ and $[\mathrm{Fe} / \mathrm{H}]=0$ ). For the spectral fitting procedure, we built a grid of synthetic composite spectra (with each component shifted by its respective RV) covering the wavelength range $[3850,5500] \AA$ computed from $\mathrm{Kurucz}^{3}$ model atmospheres with the radiative-transfer code Turbospectrum [57] using atomic linelist from VALD3 [58] in the range [3850, 4200] $\AA$ and GES linelist [59] in the range $[4200,5500] \AA$. The composite spectra in this grid combine four components, estimating radii from empirical calibration based on $T_{\text {eff }} \log g$ and $[\mathrm{Fe} / \mathrm{H}]$ [60], with temperatures ranging from 4000 to $10000 \mathrm{~K}$ (with a step of $250 \mathrm{~K}$ ), convolved with a Gaussian of standard deviation of $5 \mathrm{~km} \mathrm{~s}^{-1}$. No significant broadening was detected beyond the instrumental one. The best composite synthetic spectrum was retained on the basis of the smallest standard deviation of the (observed - calculated) residuals. The adopted $T_{\text {eff }}$ of each component is the average of the values obtained from the two spectra quoted above. The $T_{\text {eff }}$ uncertainties were computed by adding quadratically the error on the mean (since the $T_{\text {eff }}$ determination was performed on two spectra, providing slightly different results for the $C$ and $D$ components arising from the small differences between the synthetic and observed spectra at different orbital phases) and the grid step. As an illustration of the quality of the fit, Fig. 1 compares the spectrum observed on December 31, 2018 with the best combined synthetic spectrum and its individual components. The spectral types were then derived [61] from the individual temperatures of each component. We selected three PARSEC [24] isochrones with

${ }^{3}$ http: / / kurucz.harvard.edu/grids.html, the ap00k2. dat in the GRIDP00 directory 
solar metallicity $(Z=0.0147)$ covering the uncertainty range around the age of the parent cluster [19], namely $t=43^{+15}$ My. Combining these isochrones with the previously determined temperatures, the masses and luminosities of the components are derived, with uncertainties on these values deduced from the uncertainties on the component temperatures and cluster age. Radii are also derived. Finally, the spectroscopic mass ratios for each pair and for the AB-CD system, i.e. $M_{\mathrm{CD}} / M_{\mathrm{AB}}$, are derived. The astrophysical parameters of HD 74438's individual components are listed in Table 1. The large temperature range from the Gaia-ESO Survey $(7600 \pm 750 \mathrm{~K})$ well brackets the temperatures of the brightest-pair components while the Gaia temperature [70] $(7423 \pm 52 \mathrm{~K})$ agrees well with component $\mathrm{B}$.

Inclinations and separations. Combining orbital and astrophysical parameters, it is possible to deduce the inclination of each SB2 pair:

$$
i_{\mathrm{SB} 2}=\arcsin \left(\left[\frac{1}{2 \pi G}\right]^{1 / 3} K_{\mathrm{p}}\left[\frac{P}{M_{\mathrm{p}}}\right]^{1 / 3}\left[1-e^{2}\right]^{1 / 2} \frac{(1+q)^{2 / 3}}{q}\right)
$$

where $K_{\mathrm{p}}, P, M \mathrm{p}$, e and $q$ are the RV amplitude of the primary, the orbital period, the mass of the primary of the pair, the eccentricity and the mass ratio of the secondary over the primary. We obtain $i_{\mathrm{AB}}=52.5 \pm 1.5^{\circ}$ and $i_{\mathrm{CD}}=84.0 \pm 0.9^{\circ}$. Knowing the inclinations, it is then possible to derive the geometrical separation of each pair:

$$
a_{\mathrm{SB} 2}=\frac{1}{2 \pi}\left(1-e^{2}\right)^{1 / 2} P K_{\mathrm{p}} \frac{1+q}{q \sin i}
$$

where the $p$ index stands for the primary of each pair. We obtain $a_{A B}=0.21 \pm 0.25$ au and $a_{C D}=$ $0.068 \pm 0.004 \mathrm{au}$, where uncertainties on inclination dominate.

Location in the Hertzsprung-Russell (HR) diagram. The luminosity of HD 77438 (considered as a single object) is $15.3_{-0.1}{ }^{+0.2} L_{\odot}$ according to the Gaia DR2 catalogue [11]. We now compare this luminosity to the one obtained when adding the four component luminosities. Since the temperatures of the four components have been spectroscopically determined, the cluster PARSEC isochrone at 43 My directly provides their luminosities. Summing up these luminosities leads to a total luminosity of $15.7 \pm 1.8 \mathrm{~L}$ 。 for the combined system. It is represented by a horizontal line in Fig. 2, because the temperature of the SB4 considered as a single star (for instance as given by the GES temperature of $7600 \pm 750 \mathrm{~K}$; blue shaded area in Fig. 2) has no physical meaning. The combined luminosity is thus in excellent agreement with the Gaia DR2 one. The above value can be compared to the luminosity also derived from the Gaia DR2 catalogue $[11,18]$, but dereddening the photometry, using the usual relation:

$$
L=10^{-0.4\left(G+5 \log \varpi+B C_{G}-A_{G}-\mathcal{M}_{\mathrm{bol}}-10\right)} L_{\odot}
$$

where $G, \varpi, B C_{G}$, and $A_{G}$ are the Gaia $G$-band magnitude $(G=7.501 \pm 0.005)$, the parallax in mas $(\varpi=6.951 \pm 0.062)$, the bolometric correction and extinction in the $G$ band of HD 74438, respectively. The solar bolometric luminosity is taken as $\mathcal{M}_{\text {bol }}=4.75$. The extinction in the $G$ band depends on $G_{B P}-G_{R P}$ and on the reddening for IC 2391 estimated as $E(B-V)=0.100$ [11]. It is computed using Eq. 1 and Table 1 of the Gaia Collaboration [18]: $A_{G}=0.281^{+0.058}{ }_{-0.075}$ mag. 
The bolometric correction of HD 74438 is computed by considering the two most luminous components ( $A$ and $B)$ :

$$
B C_{G} \approx B C_{\mathrm{AB}}^{G}=B C_{\mathrm{A}}^{G}-2.5 \log \frac{1+L_{\mathrm{B}} / L_{\mathrm{A}}}{1+10^{-0.4\left(B C_{\mathrm{A}}^{G}-B C_{\mathrm{B}}^{G}\right)} L_{\mathrm{B}} / L_{\mathrm{A}}}
$$

where $B C_{A B}{ }^{G}, B C_{A}{ }^{G}$ and $B C_{B}{ }^{G}$ are the bolometric corrections in the $G$ band of pair $A B$, and of components $A$ and $B$ respectively. We found $B C_{A}{ }^{G}=-0.018, B C_{B}{ }^{G}=+0.061$ and $B C_{A B}{ }^{G}=+0.010$ \pm 0.013 . The bolometric correction model $B C_{G}\left(T_{\text {eff }}\right)$ in the $G$ band is computed using Eq. 7 and Table 4 from [62]. We finally obtain from Eq. (3) an extinction-corrected Gaia luminosity of $L=$ $21.1 \pm 9.5 \mathrm{~L}_{\odot}$, as represented in Fig. 2 by the brown dot. The uncertainty on the derived luminosity is obtained by propagating uncertainties from $G, \varpi$, and $B C_{G}$ and is admittedly large, because of the uncertainty on the bolometric correction. The cluster stars whose membership probability is higher than $90 \%$ are also represented as grey dots in Fig. 2, with effective temperatures as provided by the GES iDR4 [19].

Parameters of the outer orbit. We retrieved observations with GIRAFFE spectrograph taken in 2004. Combined with our recent follow-up with HRS/SALT and HERCULES/UCMJO, this allows us to cover more than half of the orbital phase. We computed the center of mass velocity of each inner pair and fit a Keplerian orbit. The fitted RV curve is given in Fig. 9. We obtained a period of about $5.7 \mathrm{y}$. The dynamical stability of hierarchical systems imposes the outer period to be at least approximately five times longer than the longest inner period [30], which is clearly the case here. The high eccentricity is not surprising for such a young system. For the derivation of the inclination of the outer pair, we used Eq. (1) while for the semi-major axis, we directly used the third Kepler law, the values being reported in Table 1.

Dynamical masses. An independent way to derive the stellar masses (besides the location of the stars in the HR diagram) resorts to the velocity semi-amplitudes through the set of equations:

$$
\frac{M_{A}}{M_{B}}=\frac{K_{A}}{K_{B}} \quad \frac{M_{C}}{M_{D}}=\frac{K_{D}}{K_{C}} \quad \frac{M_{A}+M_{B}}{M_{C}+M_{D}}=\frac{K_{C D}}{K_{A B}}
$$

The third relation leads to

$$
M_{C}=\frac{K_{A B}}{K_{C D}} \frac{1+K_{C} / K_{D}}{1+K_{A} / K_{B}} M_{A}
$$

which allows to derive three masses once one has been fixed. Adopting the lower possible spectroscopic mass value $M_{A}$ (i.e. derived from the location in the HR diagram; Table 1, row $M$ (a)), we obtained dynamical masses consistent with the spectroscopic ones for all the components. The masses derived in this way are listed in Table 1 in row $M$ (b). Because both subsystems are SB2, the dynamical mass ratios can be derived as $q=M_{\mathrm{s}} / M_{\mathrm{p}}=K_{\mathrm{p}} / K_{\mathrm{s}}$, where $\mathrm{s}$ and $\mathrm{p}$ denote the secondary and primary components, and $K$ is the RV amplitude.

Astrometric constraints. Astrometry from Gaia DR2 [11], Gaia eDR3 [12], Tycho-1 [13] and Tycho-2 [14] provide constraints on the orientation on the sky of the relative orbit $A B-C D$, as we now show. As may be seen in the $\Delta \mu_{\alpha}{ }^{*}$ and $\Delta \mu_{\delta}$ rows of Table 3, the differential proper motion of the photocentre of HD 74438 with respect to the centre of mass of the cluster varies 
according to both the epoch of the observations and the time span covered by the position observations used to derive the proper motion. That differential motion is compatible with zero for the Tycho-2 data since these long time-span data (about one century-long) average out the AB-CD orbital motion occurring on a much shorter time scale (5.7 y, as indicated in Table 1). On the contrary, the much shorter time spans of the Tycho-1, Gaia DR2 and eDR3 proper motions reveal the orbital motion on the sky. This motion is easily modelled by the Thiele-Ines constants [63] $A, B, F, G$, which allow to express the position $(x, y)$ of a component on the sky with respect to a reference point (both will be specified below) as follows:

$y=\Delta \delta \quad=A x^{\prime}+F y^{\prime}$

$x=\Delta \alpha \cos \delta=B x^{\prime}+G y^{\prime}$

with $\left(x^{\prime}, y^{\prime}\right)$ the coordinates in the orbital plane:

$x^{\prime}=r / a \cos v=\cos E-e$,

$y^{\prime}=r / a \sin v=\left(1-e^{2}\right)^{1 / 2} \sin E$,

and $v$ is the true anomaly, $E$ the eccentric anomaly, $e$ the orbital eccentricity, $r$ the length of the radius-vector and a the semi-major axis (whose exact definition will be specified below). After derivating the above relations with respect to time, the proper motion components write

$\mu_{\alpha}{ }^{*}=\mathrm{dx} / \mathrm{dt} \quad=n a^{\prime \prime}\left(B^{\prime} \frac{d x^{\prime}}{d t}+G^{\prime} \frac{d y^{\prime}}{d t}\right)=n a^{\prime \prime}\left(-B^{\prime} \frac{\sin E}{1-e \cos E}+G^{\prime}\left(1-e^{2}\right) \frac{\cos E}{1-e \cos E}\right)$

$\mu_{\delta}=\mathrm{dy} / \mathrm{dt}=n a^{\prime \prime}\left(A^{\prime} \frac{d x^{\prime}}{d t}+F^{\prime} \frac{d y^{\prime}}{d t}\right)=n a^{\prime \prime}\left(-A^{\prime} \frac{\sin E}{1-e \cos E}+F^{\prime}\left(1-e^{2}\right)^{1 / 2} \frac{\cos E}{1-e \cos E}\right)$,

with

$\dot{\mathrm{x}}^{\prime}=-\frac{\sin E}{1-e \cos E}$

$\dot{y}^{\prime}=\left(1-e^{2}\right)^{1 / 2} \frac{\cos E}{1-e \cos E}$

$A^{\prime}=(\cos \omega \cos \Omega-\sin \omega \sin \Omega \cos i)$

$B^{\prime}=(\cos \omega \sin \Omega-\sin \omega \cos \Omega \cos i)$

$F^{\prime}=(-\sin \omega \cos \Omega-\cos \omega \sin \Omega \cos i)$

$G^{\prime}=(-\sin \omega \sin \Omega+\cos \omega \cos \Omega \cos i)$

being the Thiele-Ines constants divided by $n$ a", where $n=2 \pi / P$ and $a$ " is the orbital semi-major axis expressed in arcsec, i.e., $a "=a_{a u} \dot{\omega}$ where $\dot{\omega}$ is the parallax in arcsec. The angles $\omega$ and $\Omega$ are the argument of periastron and longitude of the ascending node, respectively. In the following, since we are dealing with astrometric and spectroscopic orbits, 
these angles refer to the absolute orbit of the considered component (or photocentre) around the centre of mass of the considered system.

To go further, it is thus necessary to specify that the orbit probed by the observed differential proper motion is that of the photocentre of the AB-CD pair around the centre of mass of the system. The semi-major axis of the corresponding photocentric orbit $\left(a_{\text {phot }}{ }^{A B-C D}\right)$ is related to that of the relative $A B-C D$ orbit $\left(a_{\text {rel }}\right)$ by the relation [64]:

$a_{\text {phot }}{ }^{A B-C D}=a_{\text {rel }}(K-\beta)$,

with $\mathrm{K}=\frac{M_{C D}}{M_{A B}+M_{C D}}$

and $1 / \beta=1+10^{0.4 \Delta m}=1+\frac{L_{A B}}{L_{C D}} \quad$ where $\Delta \mathrm{m}=\mathrm{m}_{\mathrm{CD}}-\mathrm{m}_{\mathrm{AB}} \geq 0$. In the specific case of $\mathrm{HD} 74438$, $a_{r e l}=a_{A B}+a_{C D}=\left(M_{A B}+M_{C D}\right)^{1 / 3} P^{2 / 3}=5.51 \mathrm{au}, k=0.41$, and $\beta=0.0872$. Equation (8) may be converted successively in a relation for the apparent semi-major axis on the sky $\left(\mathrm{a}\right.$ " ${ }_{\text {phot }}{ }^{\mathrm{AB}-\mathrm{CD}}=$ $\left.\mathrm{a}_{\text {phot }}{ }^{\mathrm{AB}-C D} \dot{\omega}\right)$ and in a relation for the relative orbit proper motion $\left(\mu_{\text {rel }}=\mathrm{v}_{\text {rel }}=2 \pi \mathrm{a}{ }_{\text {rel }} / \mathrm{P}=\mathrm{n}\right.$

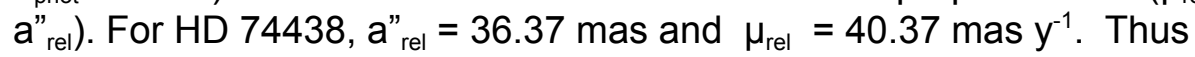

$\mu_{\text {phot }}{ }^{A B-C D}=\mu_{\text {rel }}(K-\beta)=13.03$ mas $y^{-1}$.

From the above considerations, it results that the proper-motion components observed by the astrometric satellites (and listed in Table 3) correspond to those of the photocentre of the AB-CD pair around the centre of mass of the system, i.e., $\mu_{\text {phot }}{ }^{A B-C D}$, so that the value of a" entering Eqs. $(7 a-b)$ is actually a" ${ }_{\text {phot }}{ }^{A B-C D}$. Therefore, one may define $\dot{X} \equiv \mu_{\alpha}{ }^{*} / \mu_{\text {phot }}{ }^{A B-C D}$ and $\dot{Y} \equiv \mu_{\delta} / \mu_{\text {phot }}{ }^{A B-C D}$ [with three possible pairs for $\left(\mu_{\alpha}{ }^{*}, \mu_{\delta}\right)$; namely from Tycho-1, Gaia DR2, and Gaia eDR3] such that Eqs. (7a-b) may be rewritten, after some algebra:

$\dot{X}=B^{\prime} \dot{x}^{\prime}+G^{\prime} \dot{y}^{\prime}=d \xi / d t \sin \Omega+d \eta / d t \cos i \cos \Omega$

$\dot{Y}=A^{\prime} \dot{x}^{\prime}+F^{\prime} \dot{y}^{\prime}=d \eta / d t \cos \Omega-d \eta / d t \cos i \sin \Omega$,

with

$\mathrm{d} \xi / \mathrm{dt}=\cos \omega \dot{x}^{\prime}-\sin \omega \dot{y}^{\prime}$

$\mathrm{d \eta} / \mathrm{dt}=\sin \omega \dot{x}^{\prime}+\cos \omega \dot{y}^{\prime}$.

Since these various quantities are related through a rotation, it is easy to verify that

$\dot{X}^{2}+\dot{Y}^{2}=(d \xi / d t)^{2}+(d \eta / d t)^{2} \cos ^{2} i$

and

$\left(\dot{x}^{\prime}\right)^{2}+\left(\dot{y}^{\prime}\right)^{2}=(d \xi / d t)^{2}+(d \eta / d t)^{2}$.

The quantities $\dot{X}$ and $\dot{Y}$ are proper-motion observables, obtained at some given epoch, whereas $\mathrm{d} \xi / \mathrm{dt}$ and $\mathrm{d} \eta / \mathrm{dt}$, as well as $\dot{x}^{\prime}$ and $\dot{y}^{\prime}$, depend on time through the orbital motion. Equations (9a-b) 
then allow to derive $\Omega$. Since the Gaia DR2 proper motion is the one obtained on the shortest time span among all those listed in Table 3, it most accurately represents the orbital motion and will be used in the astrometric analysis. The orbital ephemeris obtained with the orbital elements listed in Table 1 (especially $\omega=11^{\circ}$ and $i=73^{\circ}$ ) predicts $\dot{X}^{2}+\dot{Y}^{2}=0.698$ at temporal phase 0.054 and epoch JD 2457133 or April 20, 2015 (2015.3), in very good agreement with the mean epoch of Gaia DR2. At that time, the ephemeris predicts $d \xi / d t=-0.671$ and $(\mathrm{dn} / \mathrm{dt}) \cos \mathrm{i}=0.497$. From these values and the above-mentioned $\dot{X}, \dot{Y}$, Equations $(9 a-b)$ then yield $\Omega=333^{\circ}$ for the longitude of the ascending node of the orbit of the $A B-C D$ photocentre (close to that of $A B$ ) around the $A B-C D$ centre of mass. The possible operation of the ZLK phenomenon depends on the mutual orbital inclinations $\Phi$ (of the long orbit with respect to any of the short orbits). The angle $\Phi$ satisfies the relation

$\cos \Phi=\cos i_{1} \cos i_{2}+\sin i_{1} \sin i_{2} \cos \left(\Omega_{1}-\Omega_{2}\right)$

where $i_{1}, i_{2}$ are the inclinations of the long- and short-period orbit on the sky, and $\Omega_{1}, \Omega_{2}$ their respective longitudes of the ascending nodes. A definite evaluation of $\Phi$ is not possible, however, in the absence of the knowledge of the longitude of the ascending node of the short orbit. Nevertheless, given the large eccentricity $(e=0.15)$ for this short-period system $(P=4.4 d$; compare its location in the e - $\mathrm{P}$ diagram of similar systems with GV primaries in the bottom-right panel of Fig. 3), it seems likely that the ZLK phenomenon is at work through the gravitational interaction of the $C D$ pair with $A B-C D$.

Birth scenario discussion. Multiple stars are thought to be produced according to the following scenarios: (i) cluster fragmentation [65], i.e. core fragmentation for the outer (large period) subsystem and disk fragmentation for the inner ones (namely the 2 shorter period pairs) or (ii) dynamical and resonant capture. Several observables of HD 74438 contradict the capture scenarios. Some simulations of dynamical evolution within young clusters in the Solar Neighborhood have initial conditions $(N=182$, simulation time $=50$ My [66]) that match well with our case (IC 2391, with 254 objects, is 43 My old). These simulations, including primordial single, binary, triple but not quadruple stars, under-produce the latter compared to the observed fractions by a factor of three, suggesting that a primordial population of quadruples must be present. Moreover, some magneto-hydrodynamics simulations coupled with $N$-body, stellar evolution and binary interaction codes [67] show that the binary fraction drops far below the observed statistics for low-mass stars, presumably because primordial binary formation was not included. These results tend to support a formation of lower-mass multiple systems like HD 74438 predominantly by primordial core and disk fragmentations rather than by capture within the cluster.

ZLK oscillation timescales. To zeroth-order approximation, the ZLK oscillation timescale depends on the masses, the periods and the eccentricity of the outer period. Using Eq. 1 of [68] we estimated these timescales to be about $240 \mathrm{y}$ for the pair $A B$ around the center of mass of $C D$ and to be $620 \mathrm{y}$ for the pair $C D$ around the center of mass of $A B$. These correspond to $10^{4}$ to $10^{5}$ secular ZLK oscillation timescales since the birth of the cluster, enough to allow interactions (triple common envelope evolution, merger, ejection, etc.) to occur.

Relativistic precession. Depending on the system parameters, general relativity effects may cause pericenter precession, preventing the inner pair from reaching high eccentricities and thereby avoiding close encounters. We estimated the relativistic timescales $(\sim 0.1$ and $\sim 0.01 \mathrm{My}$ 
for the $A B$ and $C D$ orbit, respectively) to be much longer than the ZLK time scales quoted above. Therefore, relativistic precession does not decrease the maximum eccentricity reachable by the two inner pairs in HD 74438.

Future evolution of HD 74438. The MSE code models the evolution of multiple-star systems of arbitrary configurations [15], taking into account gravitational dynamics, stellar evolution, and binary interactions such as mass transfer and CE evolution. Also included are simple recipes for 'triple' interactions such as triple CE evolution, when a tight binary star enters the envelope of a giant star. We employ Monte Carlo methods to sample a set of realisations of the observed system taking into account the observational uncertainties. Specifically, for each system we sample all parameters (the four masses and five orbital elements for each of the three orbits, i.e., 19 parameters in total) from Gaussian distributions centred at the observed values, and with standard deviations given by the observed error bars. An exception to the latter applies to the longitudes of the ascending nodes, $\Omega_{\mathrm{i}}$, which are not all observationally constrained and which were sampled from flat distributions. With this Monte Carlo approach, we sample $N_{\mathrm{MC}}=10^{4}$ systems and evolve them with MSE until a system age of $10 \mathrm{~Gy}$ was reached, or until the maximum allowed CPU wall time of $20 \mathrm{hr}$ was reached, which occurred for about $67 \%$ of the systems. Given the highly compact nature of HD74438, the system is computationally prohibitively expensive to evolve (even using the secular approach in MSE), since the secular time-scales are short compared to $10 \mathrm{~Gy}$. Most first mergers occur at an early age of $\sim 10-100$ My, significantly younger than the attained age of most of the systems for which the CPU wall time was exceeded (peaking near $10^{9} \mathrm{y}$, see Fig. 10). This justifies the imposed maximum wall time. A summary of our results is given in Table 4, which shows the fractions per occurring physical processes, number of remnants and outcomes of our simulations. We distinguish between different events, i.e., each system can be attributed one or more events, and where we distinguish between non interacting systems, those with one or more mergers (including both CE evolution and direct collisions), CE evolution, direct collisions, dynamical instability, and RLOF of a tertiary star onto an inner binary. In the latter case, mass transfer can proceed stably (not likely), or unstably leading to triple CE evolution. About half of realisations do not lead to interactions such as mergers, whereas the other half involves interactions such as stellar mergers, and triple CE evolution. The high fraction of triple Roche Lobe Overflow (RLOF) and triple CE (close to $40 \%$ ) is much higher compared to the expectation for the entire population of isolated triple systems [69]. We classify the final outcome of the system in terms of the number of remaining objects (which can be stars or compact objects), as well as in terms of their hierarchical configuration. The non-interacting systems correspond to stable quadruple systems (fraction 0.5), which are the only final configuration with four remnants (e.g., the 'Triple+Single' outcome does not occur). When three remnants remain (fraction $\sim 0.1$ ), they can be either in a stable triple (likely), or a binary with an unbound single (not likely). If there are two remnants (fraction 0.1), the fractions of them being bound and unbound to each other are $\sim 0.03$ and $\sim 0.06$, respectively. A single remnant occurs for a fraction of $\sim 0.2$ of realisations, whereas there are no cases with no remaining remnants. In almost all instances when the tertiary fills its Roche lobe around an inner binary, the subsequent evolution is unstable and leads to triple CE evolution. In addition, the simulation show that there is a clear preference for interacting systems (in particular, mergers and triple CE systems) to have $i_{\mathrm{AB} / \mathrm{AB}-\mathrm{CD}}$ close to $90^{\circ}$, whereas the non-interacting systems favor initial $i_{\mathrm{AB} / \mathrm{AB}-\mathrm{CD}}$ further from $90^{\circ}$, with peaks near the smallest $i_{\mathrm{AB} / \mathrm{AB}-\mathrm{CD}}$ around $20^{\circ}$, and the largest around $120^{\circ}$, as shown in Fig. 11. This can be easily understood by noting that highly mutually inclined systems will lead to high eccentricities due to strong secular evolution [42]. The overwhelming majority ( 98\%) of first collisions indeed occur 
in orbit $\mathrm{AB}$. On the other hand, $i_{\mathrm{CD}}$ / $\mathrm{AB}-\mathrm{CD}$ shows less distinct features among the different outcomes, with the exception that non-interacting systems favor smaller $i_{\mathrm{CD} / \mathrm{AB}-\mathrm{CD}}$ compared to interacting ones (i.e., further separated from 90\%). Fig. 12 illustrates one possible future evolution of HD 74438 towards a WD with a sub-Chandrasekhar mass: the AB pair rapidly collides after 170 My because secular evolution drives eccentricity close to unity. The merger remnant, a $3.3 \mathrm{M}_{\odot}$ MS star, subsequently evolves and fills its Roche lobe around the companion binary at $540 \mathrm{My}$. The outcome of the triple CE is an unstable system in which a collision quickly occurs between the two components of the inner binary. A WD+MS binary remains. As the MS companion evolves, it fills its Roche lobe at around 2 Gy, ultimately causing a merger. The final remnant is a single $1.3 \mathrm{M}_{\odot}$ WD.

\section{Supplementary information}

The statistics of higher-order stellar systems are still very uncertain [30]. Recent large spectroscopic surveys have harvested many spectroscopic multiple systems with two and three components [70, 71] but very few [6] with four components (SB4).

Already known SB4. Quadruple systems with main-sequence components represent about 4\% of all stellar systems in the solar neighborhood. About 425 quadruples are known [28] and the $2+2$ hierarchy appears to be more observed than $3+1$ and trapezium systems. Nevertheless quadruple spectroscopic binaries are rather rare. To our knowledge, only eight SB4 were previously found, none of them in a cluster, all of them dimmer than HD 74438, and all of them showing eclipses. Only two of them (6. and 8.) have reached the degree of characterization of HD74438, but apparently without ZLK cycles at work. We report them below by chronological order of characterisation:

1. $B D-22^{\circ} 5866: K$ and $M$ binaries with $V=10.4$. The $K$ binary is also an $E B$ with a $2.2 \mathrm{~d}$ period. No spectral decomposition [72]; unknown inclinations.

2. V994 Her: SB4 composed of 2 pairs of co-planar eclipsing binaries: (B8V+AOV) and (A2V+A4V) with 2.1 and $1.4 \mathrm{~d}$ periods [73].

3. KIC 4247791: SB4 system with two eclipsing binaries made of 4 F-type stars (F0, F2, $F 7, F 8$ ) with $4 \mathrm{~d}$ periods for each $\mathrm{EB}$ and $\mathrm{V}=11.6$. No spectral decomposition [74];

4. KIC 7177553: SB4 system consisting of two eccentric binaries with similar periods of about $17 \mathrm{~d}$ where at least one system is eclipsing, $\mathrm{V}=11.3$. The four components are G-type stars of similar masses [75].

5. EPIC 220204960: SB4 system with two interacting eclipsing binaries made of $4 \mathrm{M}$ stars with periods of 13-14 $\mathrm{d}$ with an outer period of about $1 \mathrm{y}$, and $\mathrm{V}=12.7$ [76].

6. V482 Per: SB4 system (B9, A1, A7, A7) with 2 EB with 2.4 and $6 \mathrm{~d}$ with an outer period of $16.6 \mathrm{y}$, and $\mathrm{V}=10.3$ [77].

7. VW LMi: SB4 system (F-G spectral types) which is the tightest quadruple system with $2+2$ hierarchy yet discovered, with $0.48,7.93$ and $355 \mathrm{~d}$ periods and $\mathrm{V}=8.0$ [78].

8. CzeV1731: SB4 system with 2 twin eclipsing binaries [79].

More SB4 candidates start to emerge from large-scale spectroscopic surveys like APOGEE [80] and GALAH [81] but need follow-up observations to confirm their quadruple nature and, ultimately, characterize their orbital and astrophysical parameters. 
Other 2+2 quadruple (but not SB4) in open clusters. Our literature search (from the Multiple Star Catalogue [28] and the doubly eclipsing systems [47]) has revealed the following quadruple systems within an open cluster (OC):

- $\mu$ Ori, a member of the Hyades, is one of the brightest quadruples, with both orbits and inclinations known, as well as masses and luminosities derived from interferometry [29];

- HD 5980 (WN4+O7 I:), a member of the young OC NGC 346 in the SMC, which has been the topic of a detailed spectral analysis, but its quadruple nature is uncertain [82];

- The four other quadruples in the Hyades, as well as HD 46180 in NGC 224, and HY and KT Vel in IC 2391, have not reached the same level of characterisation as $\mu$ Ori;

- OGLE LMC-ECL-02903 is a doubly eclipsing binary in the Large Magellanic Cloud open cluster $\mathrm{KMH} 283$; the components are only characterized from the eclipse model fit using the photometric data of the OGLE survey [47].

Higher-order multiples in open clusters. Higher order multiples are difficult to discover and the ones reported in the MSC [28] mainly result from incidental findings. To date, 70 quintuples and 18 sextuples have been reported, with few of them located in OC and molecular clouds. Five septuples have also been reported with most of them being part of moving groups or associations. Due to the strong statistical biases, any conclusion on the frequency of multiples in the field and in $\mathrm{OC}$ is still premature. Large on-going and future surveys will allow us to increase the statistics of these interesting high-order multiples.

\section{References}

[53] Pasquini, L. et al., Installation and commissioning of FLAMES, the VLT Multifibre Facility, The Messenger, 110, 1 (2002) 2002Msngr.110....1P

[54] Sacco, G. G. et al., The Gaia-ESO Survey: processing FLAMES-UVES spectra, Astron. Astrophys., 565, 113 (2014) 2014A\&A...565A.113S

[55] Crawford, S. M. et al., PySALT: the SALT science pipeline, Proc. SPIE, 7737, 25 (2010) 2010SPIE.7737E..25C

[56] Kurucz, R. L., New Opacity Calculations, NATO (ASI) Series C, 341, 441 (1991) 1991ASIC..341..441K

[57] Plez, B., Turbospectrum: Code for spectral synthesis, Astrophysics Source Code Library, record ascl:1205.004

(2012) 2012ascl.soft05004P

[58] Ryabchikova, T. et al., A major upgrade of the VALD database, Phys. Scripta, 90, 5 (2015) 2015PhyS...90e4005R

[59] Heiter, U. et al., Atomic and molecular data for optical stellar spectroscopy, Phys. Scripta, 90, 5 (2015) 2015PhyS...90e4010H

[60] Moya, A., et al., Empirical Relations for the Accurate Estimation of Stellar Masses and Radii, Astrophys. J.,237, 21 (2018) 2018ApJS..237...21M

[61] Gray, R. O. \& Corbally, C. J., Stellar Spectral Classification, Princeton University Press, ISBN: 978-0-691-12511-4 (2009) 2009ssc..book.....G

[62] Andrae, R. et al., Gaia Data Release 2. First stellar parameters from Apsis, Astron. Astrophys., 616, 8, (2018) 2018A\&A...616A...8A

[63] Binnendijk, L, Properties of double stars; a survey of parallaxes and orbits, Philadelphia univ. press, (1960) 2000A\&A...355L..27H

[64] Jancart, S. et al, Astrometric orbits of $S_{B^{\wedge} 9}$ stars, Astron. Astrophys. ,442, 365 (2005) 2005A\&A...442..365J

[65] Tokovinin, A. \& Moe, M., Formation of close binaries by disc fragmentation and migration, and its statistical modelling, Mon. Not. R. Astron. Soc, 491, 5158 (2020) 2020MNRAS.491.5158T

[66] van den Berk, J., Portegies Zwart, S. F., McMillan, S. L. W., The formation of higher order hierarchical systems in star clusters, Mon. Not. R. Astron. Soc., 379, 111 (2007) 2007MNRAS.379..111V

[67] Wall, J. E., et al., Collisional N-body Dynamics Coupled to Self-gravitating Magnetohydrodynamics Reveals Dynamical Binary Formation, Astrophys. J., 887, 62, (2019) 2019ApJ...887...62W 
[68] Fabrycky, D. \& Tremaine, S., Shrinking Binary and Planetary Orbits by Kozai Cycles with Tidal Friction, Astrophys. J., 669, 1298 (2007), 2007ApJ...669.1298F

[69] Hamers, A. S. et al., A statistical view on stable and unstable Roche lobe overflow of a tertiary star onto the inner binary in triple systems, submitted to AAS Journals, 2021arXiv211000024H

[70] El-Badry, K., et al., Discovery and characterization of $3000+$ main-sequence binaries from APOGEE spectra, Mon. Not. R. Astron. Soc., 476, 528 (2018) 2018MNRAS.476..528E

[71] Traven, G. et al., The GALAH survey: Multiple stars and our Galaxy. I. A comprehensive method for deriving properties of FGK binary stars, Astron. Astrophys., 638, 145 (2020) 2020A\&A...638A.145T

[72] Shkolnik, E. et al., BD -22 5866: A Low-Mass, Quadruple-lined Spectroscopic and Eclipsing Binary, Astrophys. J., 682, 1248 2008ApJ...682.1248S

[73] Lee, C.-U., et al., V994 Herculis: the multiple system with a quadruple-lined spectrum and a double eclipsing feature, Mon. Not. R. Astron. Soc., 389, 1630 (2008) 2008MNRAS.389.1630L

[74] Lehmann, H. et al., KIC 4247791: a SB4 system with two eclipsing binaries (2EBs). A quadruple system?, Astron. Astrophys., 541, 105 (2012) 2012A\&A...541A.105L

[75] Lehmann, H. et al., KIC 7177553: A Quadruple System of Two Close Binaries, Astrophys. J., 819, 33 (2016) 2016ApJ...819...33L

[76] Rappaport, S. et al., EPIC 220204960: A Quadruple Star System Containing Two Strongly Interacting Eclipsing Binaries, 467, 2160 (2017), 2017MNRAS.467.2160R

[77] Torres, G. et al., The Quadruple-lined, Doubly Eclipsing System V482 Persei, Astrophys. J., 846, 115 (2017) 2017ApJ...846..115T

[78] Pribulla, T., et al., Secular changes in the orbits of the quadruple system VW LMi, Mon. Not. R. Astron. Soc., 494, 178 (2020), 2020MNRAS.494..178P

[79] Zasche, P. et al., CzeV1731: The unique doubly eclipsing quadruple system, Astron. Astrophys., 642, 63 (2020) 2020A\&A...642A..63Z

[80] Majweski, S.R. et al., The Apache Point Observatory Galactic Evolution Experiment (APOGEE) and its successor, APOGEE-2, Astron. Nach., 337, 863 (2016) 2016AN....337..863M

[81] De Silva, G.M. et al., The GALAH survey: scientific motivation, Mon. Not. R. Astron. Soc., 449, 2604 (2015), 2015MNRAS.449.2604D

[82] Koenigsberger, G. et al., The HD 5980 Multiple System: Masses and Evolutionary Status, Astron. J., 148,62 (2014) 2014AJ....148...62K

[83] Evans, D. S., Stars of higher multiplicity, Quarterly J. of the Royal Astron. Soc. (1968) 1968QJRAS...9..388E 
Figures
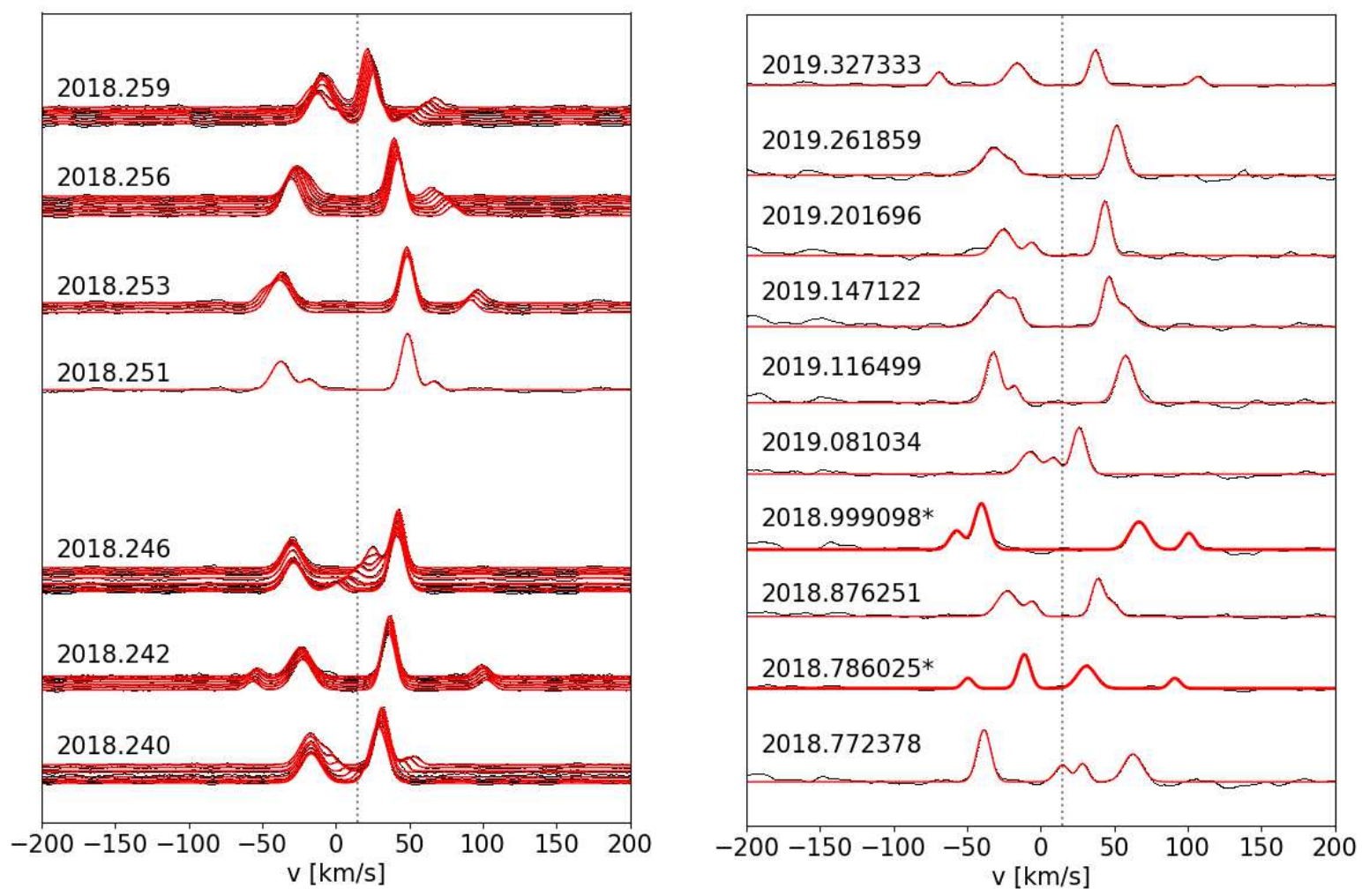

Figure 6. CCFs computed from high-resolution spectra of HD 74438 at different Julian epochs as labelled (black lines) and multi-gaussian fits (red lines). The vertical dotted line is the mean velocity of IC 2391. Left: HERCULES/UCMJO CCFs. The time series covers one week and the vertical position of the spectra scales with time. Right: same as left for HRS/SALT CCFs. The time series covers 7 months. The vertical shift of the spectra is arbitrary. Spectral fitting was performed using the two spectra marked with an asterisk and plotted in bold. 

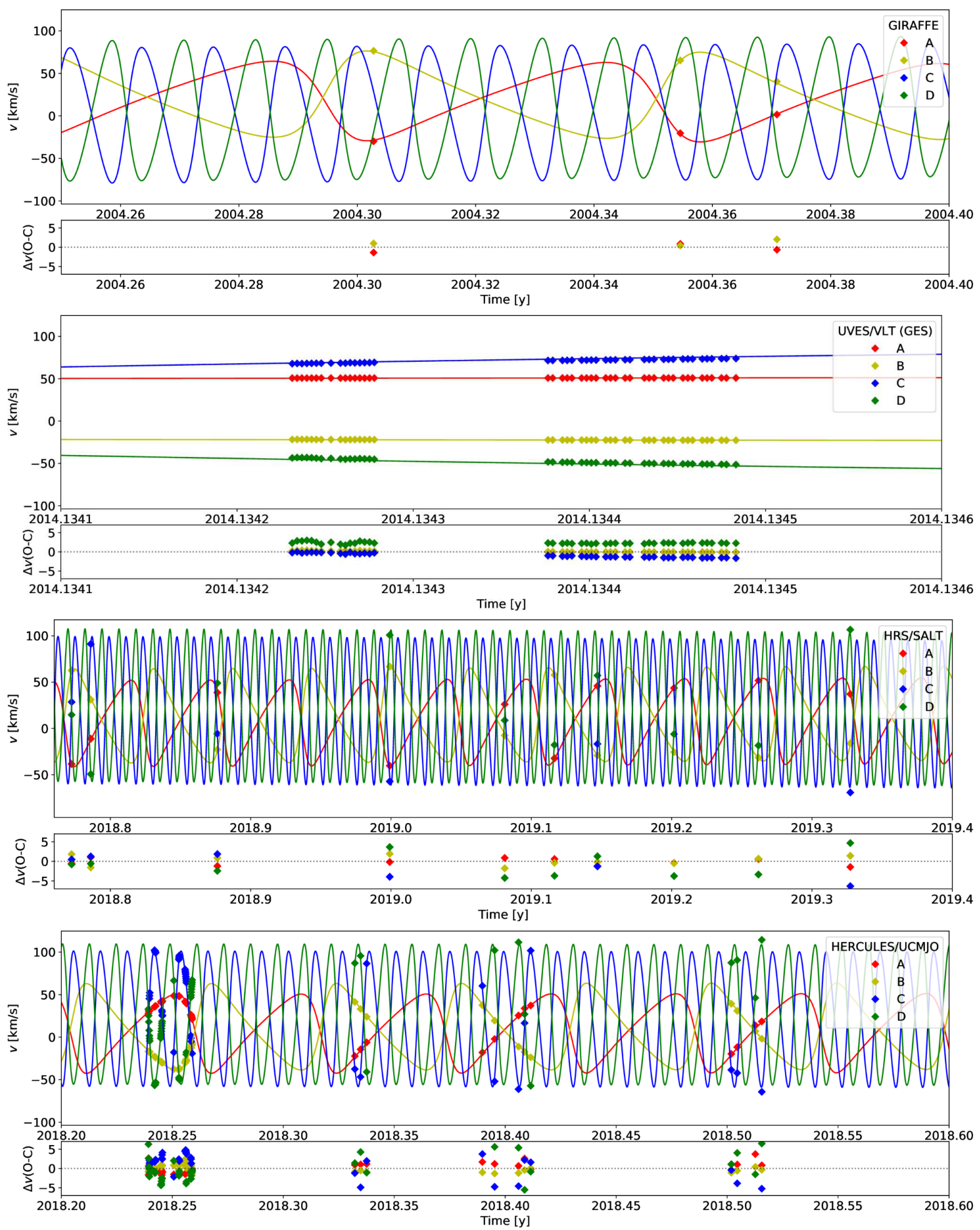

Figure 7. Orbital solutions from Table 1 and measured RVs for the four components of the SB4. Top: GIRAFFE data, middle top: UVES/VLT from GES data. middle bottom: HRS/SALT data, bottom: HERCULES/UCMJO data. Not all the RVs are presented here. 

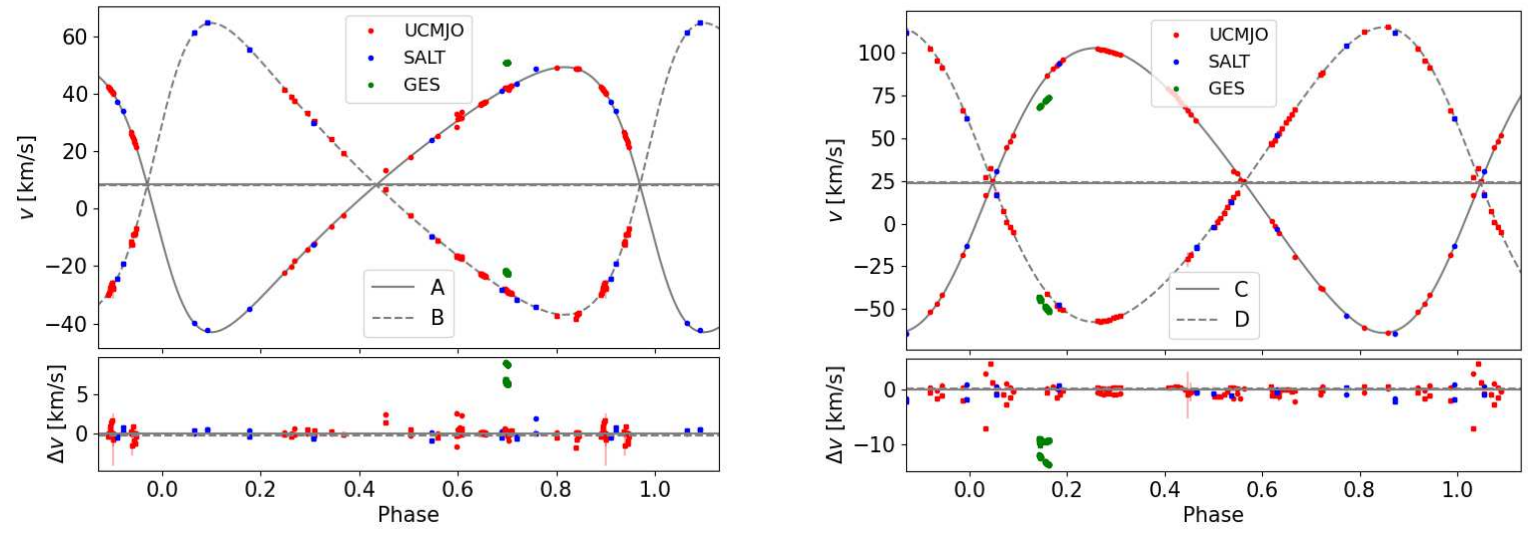

Figure 8. RV solutions using HERCULES/UCMJO and HRS/SALT data points for the brightest AB pair (left) and for the faintest CD pair (right). The GES data points are not included in the computation of the orbital solutions because taken $5 \mathrm{y}$ before, showing the gravitational effect between the two inner pairs.

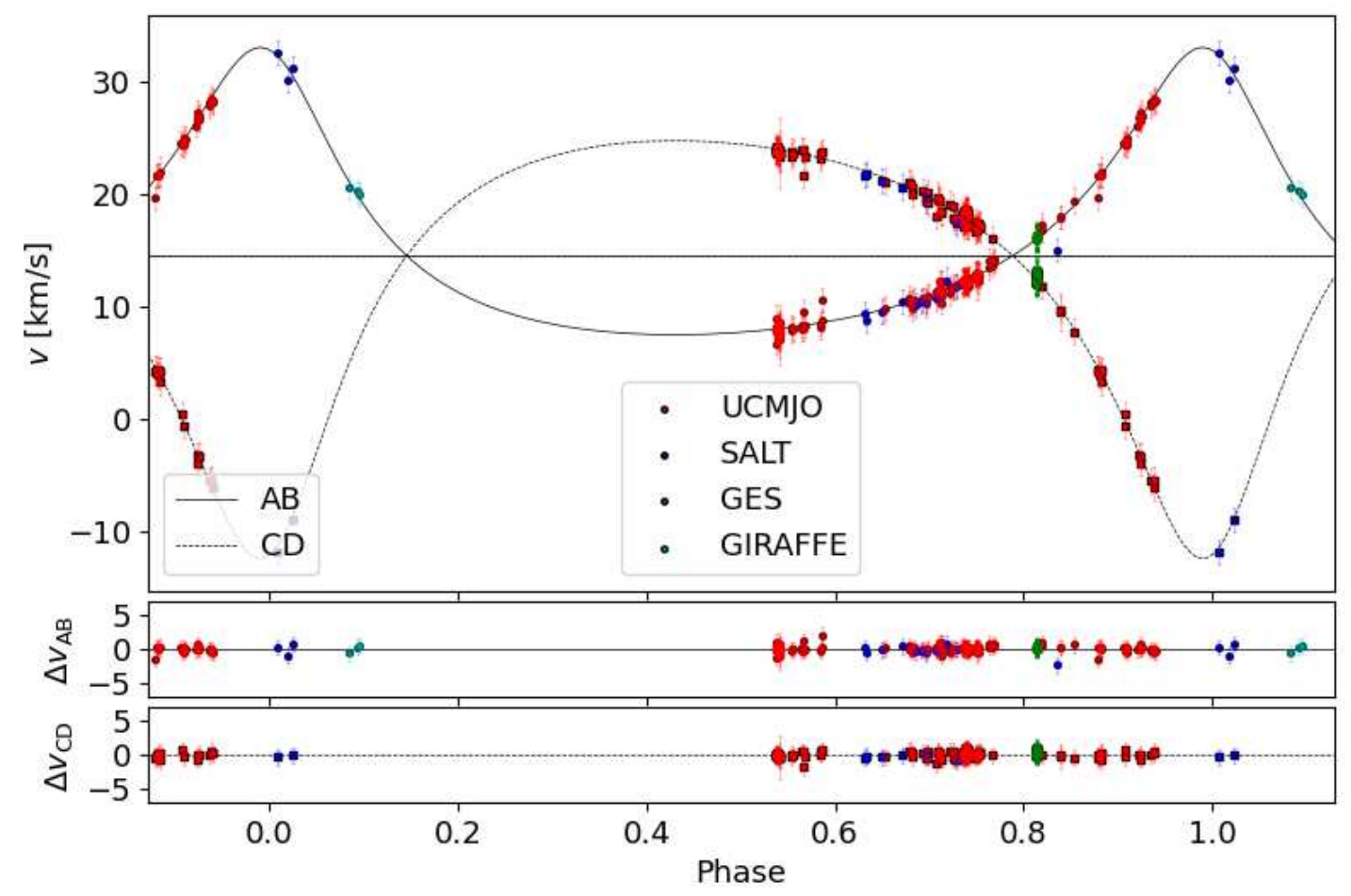

Figure 9. RV solution of the wide pair $A B-C D$ using center of mass $R V$ of the $A B$ and $C D$ pairs. 


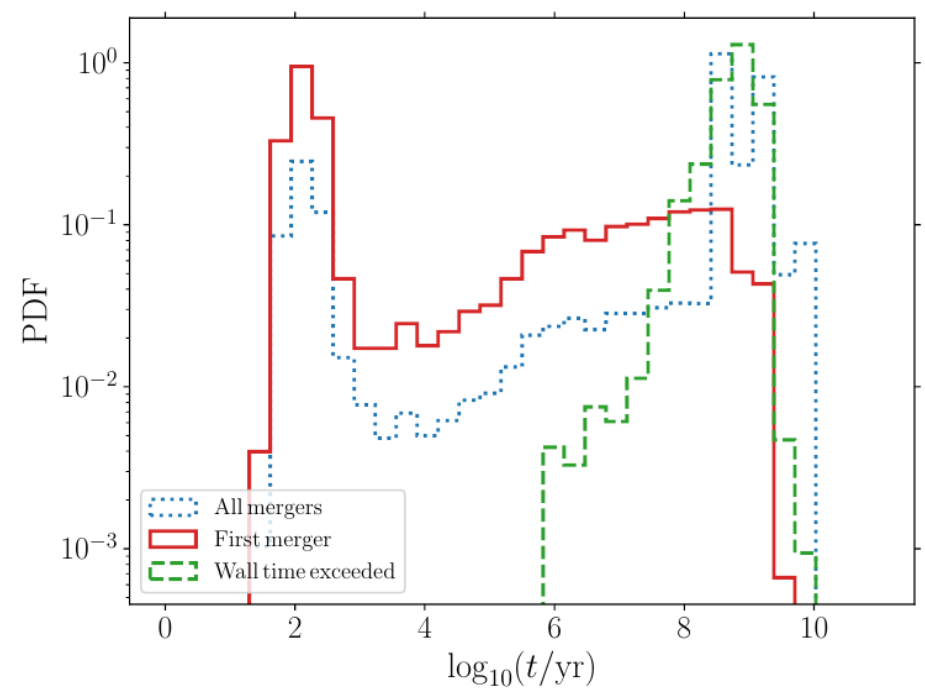

Figure 10. Probability distributions of merger times in the simulations. The distribution of times of all merger events is displayed with the blue dotted lines, and the times of the first merger in the system (if applicable) with the red solid line. The distributions of the maximum age reached by the system for the systems in which the maximum wall time was exceeded is shown with the green dashed line.
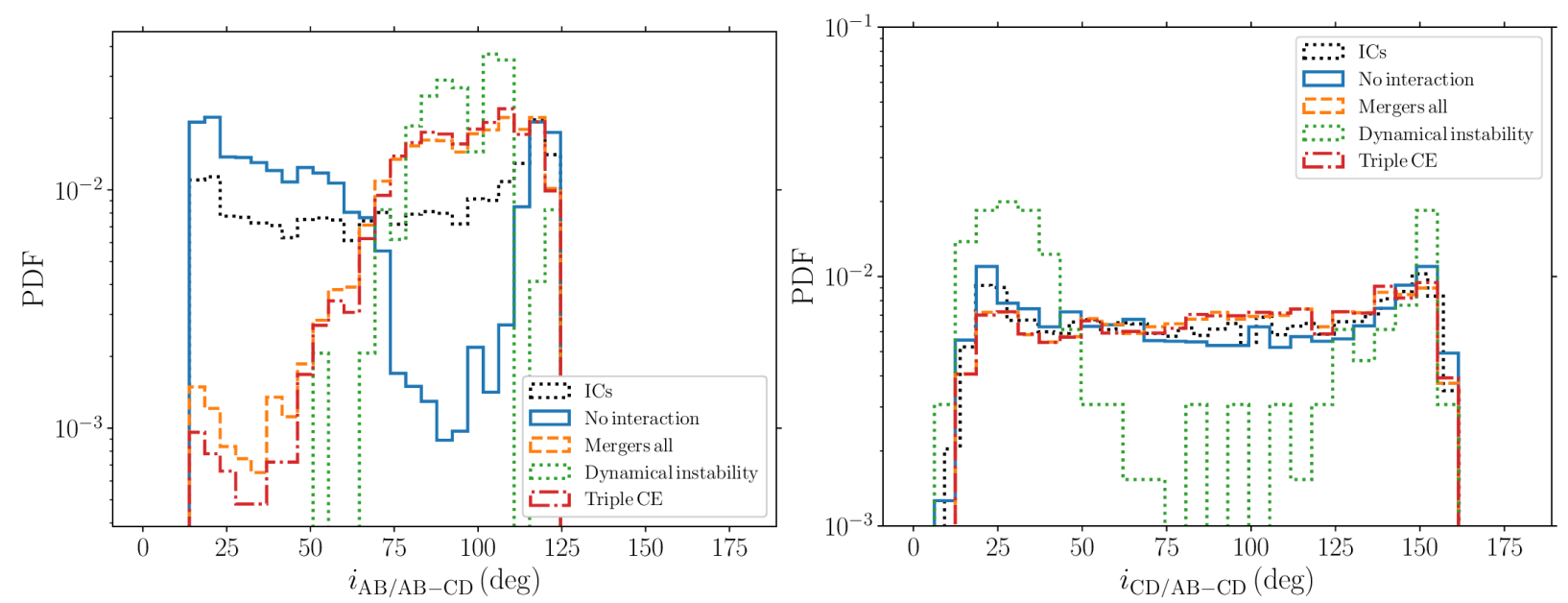

Figure 11. Probability distributions of the current mutual inclinations (left panel: $i_{\mathrm{AB} / \mathrm{AB}-\mathrm{CD}}$; right panel: $i_{\mathrm{CD} / \mathrm{AB}-\mathrm{CD}}$ ) leading to different events in our Monte Carlo simulations (as described in the legends). The curve labelled ICs corresponds to the distribution of the initial mutual inclinations (encompassing all possible outcomes). This distribution is obtained from the observed values of the individual orbital inclinations on the sky, complemented by flat distributions for the longitudes of the unknown ascending nodes (Eq. 10). 


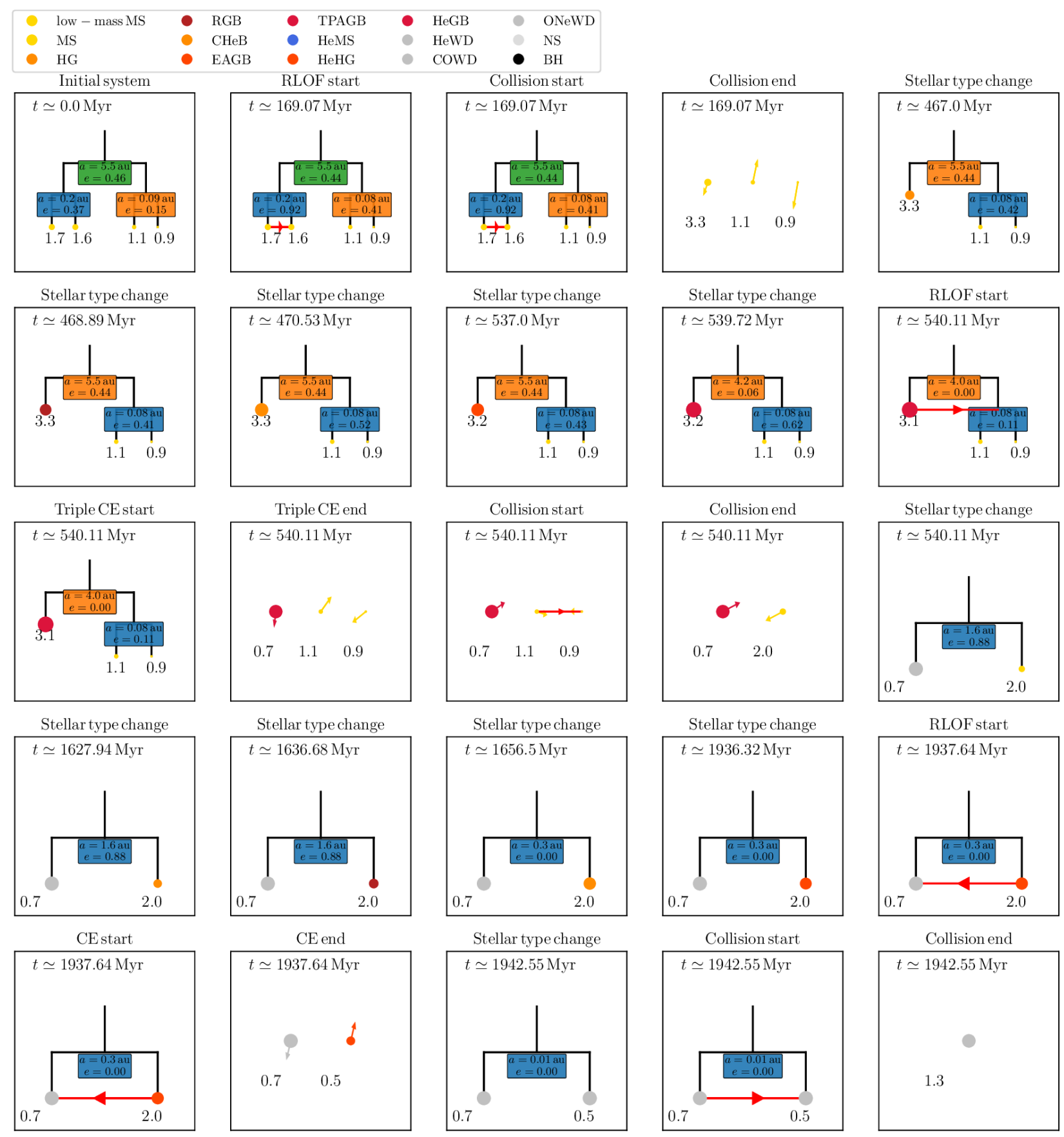

Figure 12. One possible future evolution of HD 74438. Each panel shows an event of interest as labeled at the top, with the time indicated in each subpanel and the system represented schematically in a so-called mobile diagram [83, see also 15], with orbital parameters (semimajor axes $a$ and eccentricities $e$ ) and masses (in units of $M_{\odot}$ ) indicated. The meaning of the dot colors (representing the stars) is indicated in the legend at the top. The quadruple experiences unstable RLOF, triple CE and merger events, leaving a WD remnant with a sub-Chandrasekhar mass of $1.3 \mathrm{M}_{\odot}$. 


\begin{tabular}{|c|c|c|c|c|}
\hline Instrument & $\begin{array}{c}\text { Resolving } \\
\text { power } \\
(\lambda / \Delta \lambda)\end{array}$ & $\begin{array}{c}\text { Spectral coverage } \\
(\boldsymbol{A})\end{array}$ & $\begin{array}{c}\text { Number of } \\
\text { exposures }\end{array}$ & Epoch range \\
\hline GIRAFFE/VLT & 22000 & {$[3850,4050]$} & 3 & $25 \mathrm{~d}$ \\
\hline UVES/VLT & 47000 & {$[4800,6200]$} & 45 & $2.5 \mathrm{~h}$ \\
\hline HERCULES/UCMJO & 41000 & {$[4160,7635]$} & 193 & $\sim 2.3 \mathrm{y}$ \\
\hline HRS/SALT & 65000 & {$[3830,8775]$} & 13 & $\sim 2 \mathrm{y}$ \\
\hline
\end{tabular}

Table 2. Summary of the spectroscopic observations.

\begin{tabular}{|c|c|c|c|c|c|}
\hline Catalogue & $\begin{array}{l}\text { HD } 74438 \\
\text { (Tycho-1) }\end{array}$ & $\begin{array}{l}\text { HD } 74438 \\
\text { (Tycho-2) }\end{array}$ & $\begin{array}{l}\text { HD } 74438 \\
\text { (Gaia DR2) }\end{array}$ & $\begin{array}{c}\text { HD } 74438 \\
\text { (Gaia eDR3) }\end{array}$ & $\begin{array}{l}\text { Cluster } \\
\text { center }\end{array}$ \\
\hline Mid-epoch (y) & 1991.25 & 1991.23 & 2015.5 & 2016.0 & - \\
\hline Time span (y) & 3.5 & $\sim 100$ & 1.8 & 2.8 & - \\
\hline$\mu_{\alpha}^{*}\left(\operatorname{mas} \mathrm{y}^{-1}\right)$ & $-33.5 \pm 4.0$ & $-23.4 \pm 1.5$ & $-15.895 \pm 0.122$ & $-17.74 \pm 0.09$ & $-24.93 \pm 0.08^{13}$ \\
\hline$\mu_{\delta}\left(\operatorname{mas} y^{-1}\right)$ & $+27.8 \pm 3.4$ & $+23.7 \pm 1.4$ & $+17.256 \pm 0.131$ & $+17.06 \pm 0.10$ & $+23.26 \pm 0.11^{13}$ \\
\hline$\Delta \mu_{\alpha}^{*}\left(\operatorname{mas} \mathrm{y}^{-1}\right)$ & $-8.57 \pm 4.0$ & $1.53 \pm 1.5$ & $9.03 \pm 0.14$ & $7.19 \pm 0.12$ & - \\
\hline$\Delta \mu_{\delta}\left(\operatorname{mas} y^{-1}\right)$ & $4.54 \pm 3.4$ & $0.44 \pm 1.4$ & $-6.00 \pm 0.17$ & $-6.20 \pm 0.15$ & - \\
\hline$\dot{X}=\Delta \mu_{a}{ }^{*} / \mu^{\prime \prime}{ }_{A B-C D, p h o t}$ & -0.659 & 0.118 & 0.694 & 0.553 & - \\
\hline$\dot{Y}=\Delta \mu_{\delta} / \mu^{\prime \prime}{ }_{A B-C D, p h o t}$ & 0.349 & 0.034 & -0.461 & -0.477 & - \\
\hline$\dot{X}^{2}+\dot{Y}^{2}$ & 0.556 & 0.015 & 0.694 & 0.533 & - \\
\hline
\end{tabular}

Table 3. Proper motions in right ascension (RA, including the cos De factor) and declination (De). The differential proper motion $\Delta \mu_{\alpha}{ }^{*}$ and $\Delta \mu_{\delta}$ denote the differential stellar motion with respect to the cluster. The differential proper motions from Tycho-1, Gaia DR2, and Gaia eDR3 reflect the orbital motion of the $A B C D$ photocentre around the centre of mass of the cluster. 


\begin{tabular}{|l|c|c|c|}
\hline \multicolumn{1}{|c|}{ Description } & Fraction & Final outcomes & Fraction \\
\hline No interaction & $0.535 \pm 0.007$ & Quadruple & $0.535 \pm 0.007$ \\
\hline Mergers all & $0.465 \pm 0.007$ & Single & $0.236 \pm 0.005$ \\
\hline CE & $0.298 \pm 0.005$ & Triple & $0.117 \pm 0.003$ \\
\hline Collision & $0.461 \pm 0.007$ & Two Single & $0.058 \pm 0.002$ \\
\hline Dynamical instability & $0.010 \pm 0.001$ & Binary & $0.030 \pm 0.002$ \\
\hline Triple RLOF & $0.363 \pm 0.006$ & Binary+Single & $0.024 \pm 0.002$ \\
\hline Triple CE & $0.360 \pm 0.006$ & & \\
\hline Number of final remnants & Fraction & Triple CE outcomes & Fraction \\
\hline 1 & $0.236 \pm 0.005$ & Triple & $0.036 \pm 0.003$ \\
\hline 2 & $0.088 \pm 0.003$ & Merger(s) & $0.705 \pm 0.014$ \\
\hline 3 & $0.141 \pm 0.004$ & Binary+Single & $0.152 \pm 0.006$ \\
\hline 4 & $0.535 \pm 0.007$ & Indeterminate & $0.107 \pm 0.005$ \\
\hline
\end{tabular}

Table 4. Outcome fraction per physical process (several processes can actually occur for a given simulated system), number of remnants and final outcomes of MSE simulations. Statistical error bars are given (based on Poisson statistics). 'Mergers all' means that any kind of merger occurred at any point, irrespective of whether or not an ejection event happened. Dynamical instability represents instability triggered by stellar evolution (e.g. orbital expansion due to wind mass loss). In some cases, the outcome was indeterminate from the simulation data in relation to the CPU wall time being exceeded. 